\title{
Charge and spin control of ultrafast electron and hole dynamics in single CdSe/ZnSe quantum dots
}

\author{
C. Hinz, ${ }^{1}$ P. Gumbsheimer, ${ }^{1}$ C. Traum, ${ }^{1}$ M. Holtkemper, ${ }^{2}$ B. Bauer, ${ }^{1}$ J. Haase, ${ }^{1}$ S. Mahapatra,${ }^{3}$ A. Frey, ${ }^{3}$ K. Brunner, ${ }^{3}$ \\ D. E. Reiter, ${ }^{2}$ T. Kuhn, ${ }^{2}$ D. V. Seletskiy, ${ }^{1,4}$ and A. Leitenstorfer ${ }^{1}$ \\ ${ }^{1}$ Department of Physics and Center for Applied Photonics, University of Konstanz, D-78464 Konstanz, Germany \\ ${ }^{2}$ Institut für Festkörpertheorie, Universität Münster, Wilhelm-Klemm-Straße 10, D-48149 Münster, Germany \\ ${ }^{3}$ Institute of Physics, EP 3, University of Würzburg, D-97074 Würzburg, Germany \\ ${ }^{4}$ Department of Engineering Physics, École Polytechnique de Montréal, Montréal, Quebec H3T 1J4, Canada
}

\begin{abstract}
We study the dynamics of photoexcited electrons and holes in single negatively charged CdSe/ZnSe quantum dots with two-color femtosecond pump-probe spectroscopy. An initial characterization of the energy level structure is performed at low temperatures and magnetic fields of up to $5 \mathrm{~T}$. Emission and absorption resonances are assigned to specific transitions between few-fermion states by a theoretical model based on a configuration interaction approach. To analyze the dynamics of individual charge carriers, we initialize the quantum system into excited trion states with defined energy and spin. Subsequently, the time-dependent occupation of the trion ground state is monitored by spectrally resolved differential transmission measurements. We observe subpicosecond dynamics for a hole excited to the $D$ shell. The energy dependence of this $D$-to- $S$ shell intraband transition is investigated in quantum dots of varying size. Excitation of an electron-hole pair in the respective $p$ shells leads to the formation of singlet and triplet spin configurations. Relaxation of the $p$-shell singlet is observed to occur on a time scale of a few picoseconds. Pumping of $p$-shell triplet transitions opens up two pathways with distinctly different scattering times. These processes are shown to be governed by the mixing of singlet and triplet states due to exchange interactions enabling simultaneous electron and hole spin flips. To isolate the relaxation channels, we align the spin of the residual electron by a magnetic field and employ laser pulses of defined helicity. This step provides ultrafast preparation of a fully inverted trion ground state of the quantum dot with near unity probability, enabling deterministic addition of a single photon to the probe pulse. Therefore our experiments represent a significant step towards using single quantum emitters with well-controled inversion to manipulate the photon statistics of ultrafast light pulses.
\end{abstract}

\section{INTRODUCTION}

On-demand generation of nonclassical states of light is a highly sought-after goal for tasks of quantum photonics and quantum information science. The degree of quantumness of a state, and hence its resource for quantum processing, can in general be ascertained by its deviation from a coherent state [1]. In this context, a novel class of quantum states was implemented [2,3], where nonclassicality [4] is achieved by addition/subtraction of a single photon to/from a coherent state. Such nonclassical states with manipulated photon numbers have recently been utilized for a variety of tasks, from characterization of bosonic ladder operators [5] to noiseless coherent state amplification [6]. The single-photon adder operation has been demonstrated in single quantum dots [7]. In this scheme, resonant femtosecond $\pi$-pulse excitation of a singly charged quantum dot initializes an excited three-particle state, a trion. Relaxation of the trion toward its ground state leaves the quantum dot in a state with inverted population. In the second step, the system is readout by a femtosecond $\pi$ pulse, thereby adding a single photon to the temporal mode of the probe field. Together with the generation of the nonclassical output, the important advantage of this amplifier is in its ultrafast response, where the gain bandwidth is limited only by the energetic separation of the initialization and readout transitions. Under such quantum operation of the amplifier, the spectral weight of a differential transmission signal is evenly distributed over the entire probe bandwidth. In contrast, in this work, we interrogate microscopic mechanisms governing the dynamics and selection rules of such single-photon amplifiers by studying them in the small-gain regime (pulse area $<\pi$ ). Here, the gain bandwidth remains narrow and the full spectral information is maintained. Therefore operation in the linear readout regime is more adequate for quantitative characterization of the underlying relaxation processes as well as inputoutput performance of the quantum dot-based single-photon amplifiers. Moving toward designer nonclassical states of light in the time domain is then straightforward by increasing the pulse area of the probe.

Semiconductor quantum dots (QDs) are often termed "artificial atoms" due to their discrete electronic energy levels. They represent an attractive platform for fundamental studies on quantum physics but also enable a variety of everyday applications [8-10]. Envelope wave functions of electron and hole states, which are distributed over many atomic sites lead to large dipole moments associated with excitonic excitations. Especially, trions in charged QDs [11] provide strongly dipole-allowed transitions into their ground states. This feature is attractive for quantum information technologies [12], for example, when high-speed manipulation of the spin 
degree of freedom is desired [13-17]. In this context and the context of the single-photon amplifier, it is important to understand the intrinsic relaxation mechanisms which can influence preparation and readout of the few-particle quantum states via ultrashort light pulses. To this end, charge-carrier dynamics in QDs based on III-V semiconductors with shallow quantum confinement has been studied both in large ensembles [18-20] and single specimens [7,21-26]. These developments have culminated in demonstrations of optical writing and readout of spin states in single or coupled QDs [13-17,27]. While transition metal dichalcogenide monolayers (TMDs) with large exciton binding energies have been shown to emit single photons [28-30], we are not aware of any experimental demonstrations of qubit operations despite recent theoretical analysis [31].

In this work, we investigate the elementary dynamics of electrons and holes in single CdSe/ZnSe QDs with femtosecond temporal resolution. Large confinement potentials and strong Coulomb interactions, which follow from a reduced dielectric screening, lead to charge and spin configurations with larger separations between quantized energy levels as compared to their III-V counterparts [32-35]. This fact enables ultrafast quantum manipulation with broadband laser pulses. So far, investigations of ultrafast dynamics in II-VI systems have been carried out on ensembles of QDs, usually involving excitations into the interband continuum [36-42]. In contrast, we are motivated to initialize and readout the bound states on ultrafast time scales where the discrete frequencies and well-defined spin configurations of electronic transitions offer deterministic coupling for high-fidelity quantum processing schemes. Previously, single-photon gain on the transition from the trion ground state to the Fermi vacuum of a negatively charged QD has been demonstrated [7,43]. Here we focus on the ultrafast dynamics between distinct charge and spin configurations of both electrons and holes. Our findings include a subpicosecond and polarization-selective buildup of singlephoton gain at maximum fidelity. This feature will be crucial in future efforts to control the quantum statistics of ultrafast laser pulses by direct manipulation of their photon number.

The presentation is structured in the following way: the samples consisting of single $\mathrm{CdSe} / \mathrm{ZnSe}$ QDs coupled to nanophotonic structures for enhancement of light-matter coupling are outlined in Sec. II. Also, the two-color femtosecond pump-probe setup built around a superconducting magnetic cryomicroscope is described, including a lock-in readout scheme, which avoids modulation of the pump power. Section III explains the energetic spectrum of few-particle states in negatively charged CdSe/ZnSe QDs and related interband transitions. General features of intraband dynamics leading to population inversion, i.e., formation of the trion ground state, are investigated in Sec. IV for various excitation energies. We also introduce a rate-equation model to extract the time scales underlying the dynamics which helps to reveal a twocomponent relaxation in the case of triplet-to-ground-state trion scattering. The onset of ultrafast single-photon gain is studied in Sec. V. This process contains precise information on hole relaxation and spin dependence of electron dynamics. In Sec. VI, we demonstrate the capability to maximize and control single-photon gain by application of an external magnetic field. Nearly perfect fidelity of the polarization-selective inversion is achieved following the absorption of the excitation pulse, reaching an overall photon-to-photon conversion on the order of $0.1 \%$. Coherent spin dynamics owing to Larmor precession of the trion ground state is also observed and discussed. Finally, Sec. VII provides a summary and outlook perspectives for ultrafast quantum optics.

\section{SAMPLES, EXPERIMENTAL SETUP, AND METHODOLOGY}

Motivated by possible femtosecond operation of future single-photon amplifiers, we do not employ high- $Q$ microresonators which, while highly successful for few-photon nonlinear quantum optics in the subnanosecond range [44], cannot support gain over a broad bandwidth. With this in mind, we developed a nanophotonic concept for maximum broadband coupling between an optical readout pulse and single electrons in a semiconductor quantum structure, sketched in Fig. 1(a). We investigate epitaxially grown singly charged CdSe/ZnSe QDs with a CdSe core diameter (red dots) between 3 and $6 \mathrm{~nm}$ [45]. They are enclosed in a ZnSe matrix of $100 \mathrm{~nm}$ thickness. ZnSe discs (yellow) are embedded into aluminum nanoapertures (dark grey) of 200 to $400 \mathrm{~nm}$ diameter by means of focused ion beam milling and a sequence of evaporation and lift-off steps. The resulting structures contain approximately 10 QDs, which are separable by selective spectral excitation and readout. This geometry allows us to reduce the effective diameter of the optical beams and to exploit the particular transmission properties of subwavelength apertures [46]. Diameters are chosen to match the extraordinary transmission of the structure to the emission resonances of the QD [47]. A poly(methyl methacrylate) (PMMA, light grey) pillar matching the $\mathrm{ZnSe}$ disc diameter remains on top of the nanoaperture. It serves both as a monomode waveguide and antireflection coating to effectively couple pump and probe light to the QD. Owing to the high spatial resolution of the focused ion beam milling process of less than $7 \mathrm{~nm}$, these structures can be produced with a nearly perfect circular shape, rendering them applicable for measurements involving defined helicity for pumping and readout. A quartz substrate $\left(\mathrm{SiO}_{2}\right.$, light blue $)$ of $120 \mu \mathrm{m}$ thickness allows for transmission experiments. The complete sample structure is shown in a scanning electron micrograph on the right hand side of Fig. 1(a).

Figure 1(b) depicts the experimental setup used for ultrafast analysis of intraband relaxation of few-particle states in single quantum emitters. The source of excitation (green) and probe (red) pulses is a two-branch femtosecond Er:fiber laser system [48], with each output independently tunable in central photon energy from 1.77 to $2.48 \mathrm{eV}$. A bandwidth of the pump pulses of $2 \mathrm{meV}$ in full width at half maximum (FWHM) is used in order to selectively excite interband transitions while preserving a temporal resolution of $600 \mathrm{fs}$. The pulse duration for probing is set to $100 \mathrm{fs}$, corresponding to a FWHM bandwidth of $20 \mathrm{meV}$. Probe pulses contain $10^{4}$ photons on average, corresponding to an energy of $100 \mathrm{fJ}$. Such low flux of probe photons attests to the high sensitivity of our setup and the efficient light-matter coupling achieved by the photonic structures. The time delay $t_{D}$ between pump and probe pulses is adjusted by means of a retroreflector placed on a linear translation stage in the optical path of the probe branch. In this way, 
(a)

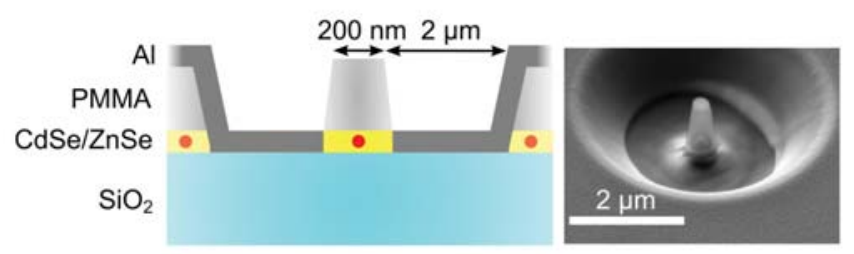

(c)

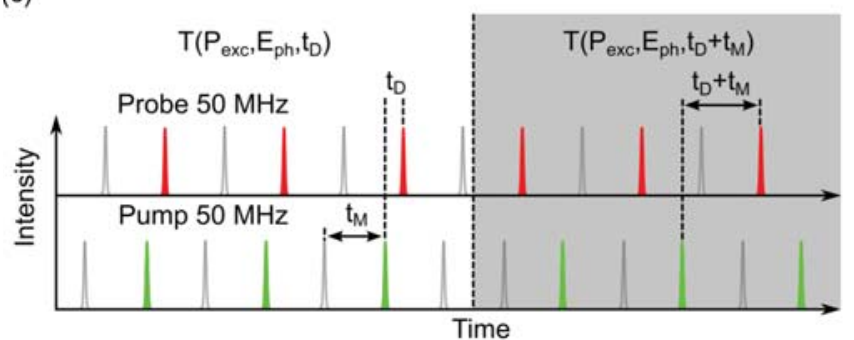

(b)

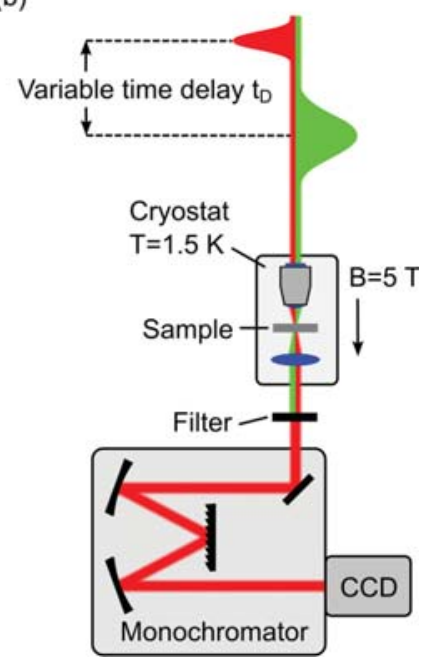

FIG. 1. (a) Left: sketch of the nanophotonic structure. Single CdSe cores (red circles) in a ZnSe layer (yellow) with a thickness of $100 \mathrm{~nm}$ are embedded into an aluminum nanoaperture (dark grey). The diameter is approximately $200 \mathrm{~nm}$. The aperture is covered by a PMMA pillar. Right: SEM micrograph of the sample. (b) Experimental setup: femtosecond pump (green) and probe (red) pulses derived from a two-color Er:fiber laser are focused onto the sample using an objective lens (grey) with $N A=0.9$. An aspheric lens (blue) with $N A=0.68$ is used for collection of the transmitted probe. Measurements take place under an optional magnetic field of $B=5 \mathrm{~T}$ and a sample temperature $T_{L}$ down to $1.5 \mathrm{~K}$ in an optical magnet cryostat. The differential transmission signals are detected using a monochromator and a cooled CCD camera. (c) Sketch of the pulse sequence for pumping and probing implemented in the experiment. The top part depicts the output of the probe branch (red) at a repetition rate of $50 \mathrm{MHz}$. The pulse train in the pump branch (green) is shown at the bottom. Pulses colored in grey are eliminated by an electro-optic modulator. $t_{M}$ corresponds to the interpulse distance at the full repetition rate of the Er:fiber laser system of $100 \mathrm{MHz}$, while $t_{D}$ is the timing between pump and probe set by a variable optical delay. This scheme allows lock-in detection of differential transmission changes of the sample without modulation of the average excitation or probing powers. Regions with white and grey background indicate the two timing phases of 15 -ms duration the lock-in amplifier is referenced to.

we can vary $t_{D}$ between -200 and $+200 \mathrm{ps}$. The samples are mounted in a superconducting cryo-magnet system that allows for transmission measurements. Experiments may be carried out at substrate temperatures down to $T_{L}=1.5 \mathrm{~K}$ and under an optional magnetic field of up to $B=5 \mathrm{~T}$ applied in Faraday geometry. For optimum coupling efficiency, we use an objective lens with numerical aperture $N A=0.9$ for excitation and a lens with $N A=0.68$ for collection of photoluminescence emission or transmitted probe photons. The probe spectrum is dispersed by a grating monochromator and detected with a cooled CCD array at a resolution of $100 \mu \mathrm{eV}$.

In our experiments, we measure the spectrally resolved normalized differential transmission $\Delta T / T$ of the probe pulses. For a fixed time delay $t_{D}$ and photon energy $E_{\mathrm{ph}}$, this quantity usually represents the normalized difference between the transmission of the probe through a quantum system $T\left(P_{\mathrm{exc}}, E_{\mathrm{ph}}, t_{D}\right)$ exposed to a certain excitation power $P_{\mathrm{exc}}$ and the transmission without excitation $T\left(0, E_{\mathrm{ph}}, t_{D}\right)$ :

$$
\frac{\Delta T}{T}=\frac{T\left(P_{\mathrm{exc}}, E_{\mathrm{ph}}, t_{D}\right)-T\left(0, E_{\mathrm{ph}}, t_{D}\right)}{T\left(0, E_{\mathrm{ph}}, t_{D}\right)} .
$$

In standard pump-probe measurements, the average excitation power $P_{\text {exc }}$ is modulated, as suggested by Eq. (1). It turns out that in experiments on a discrete quantum structure with narrow electronic resonances, this procedure can lead to significant parasitic signals associated, e.g., with periodic thermal heating or background luminescence. To eliminate such effects, we modulate the time delay $t_{D}$ by an offset $t_{M}$ while keeping $P_{\text {exc }}$ constant, thus avoiding any spurious artefacts. The definition for $\Delta T / T$ then becomes

$$
\frac{\Delta T}{T}=\frac{T\left(P_{\mathrm{exc}}, E_{\mathrm{ph}}, t_{D}\right)-T\left(P_{\mathrm{exc}}, E_{\mathrm{ph}}, t_{D}+t_{M}\right)}{T\left(P_{\mathrm{exc}}, E_{\mathrm{ph}}, t_{D}+t_{M}\right)} .
$$

The value of $t_{M}$ is chosen such that the system has returned to the ground state at a time delay $t_{D}+t_{M}$. In our studies, the temporal offset $t_{M}$ has to exceed the spontaneous emission lifetime of the quantum emitters. To implement this scheme, we insert fiber-coupled electro-optic modulators in both the pump and the probe branches of the femtosecond Er:fiber laser. The full pulse train produced by the mode-locked oscillator of the laser system operates at a repetition rate of $100 \mathrm{MHz}$ [indicated by the grey and colored maxima in Fig. 1(c)]. After the output coupler of the oscillator, a fiber-optic beam splitter separates the pulse trains that are used to seed the two parallel femtosecond Er:fiber amplifiers, which are employed for pumping and probing, respectively. The electro-optic modulators select only every second pulse out of this pulse train, effectively reducing the repetition rate to $50 \mathrm{MHz}$. There are now two different options: in the first case [left part of Fig. 1(c)], the same pulse out of the original $100 \mathrm{MHz}$ pulse train is amplified in both branches. In this case, $t_{D}$ may be fine tuned with the variable delay stage and $T\left(P_{\mathrm{exc}}, E_{\mathrm{ph}}, t_{D}\right)$ is measured. Alternatively, one pulse out of the $100 \mathrm{MHz}$ pulse train is selected in one branch and the following pulse is amplified in the other branch. As shown in the grey shaded region in the right part of Fig. 1(c), the timing between pump and probe pulses is set to $t_{D}+t_{M}$ where $t_{M}=10 \mathrm{~ns} \gg t_{D}$ is given by the interpulse distance in the original $100 \mathrm{MHz}$ pulse train. Consequently, $t_{D}+t_{M}$ by far 
(a)

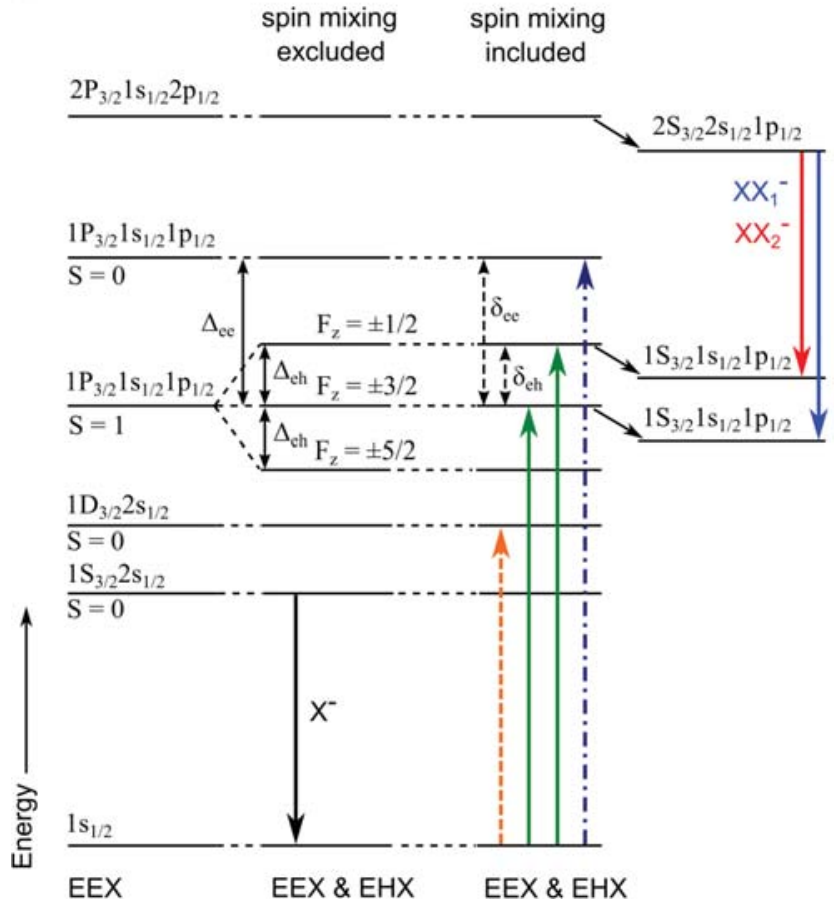

(b)
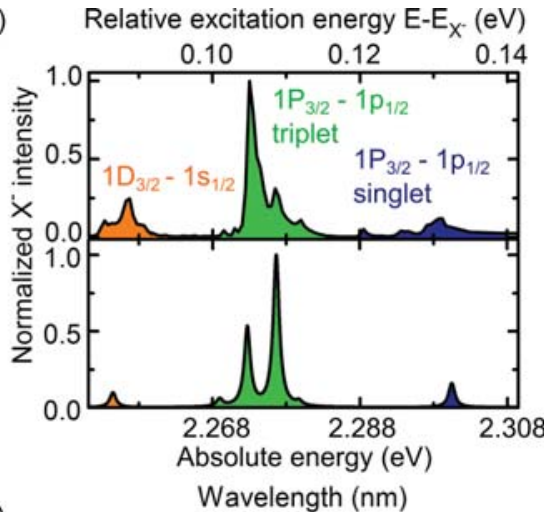

(c)

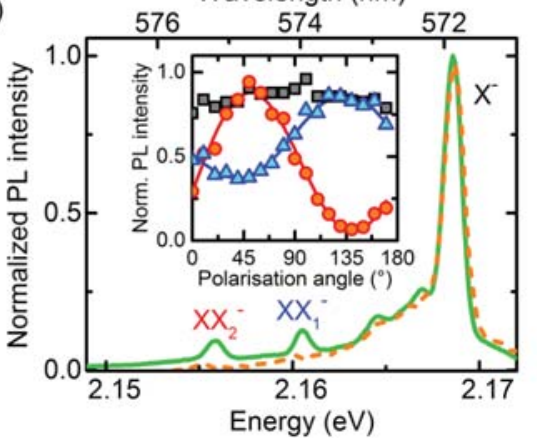

FIG. 2. (a) Schematic of the energetic structure of a negatively charged quantum dot (QD). Dipole-allowed absorption and emission transitions are shown with colored downward and upward arrows, respectively. (b) Emission strength of the fundamental trion line (label "X" ${ }^{-}, E_{\mathrm{X}^{-}}=2.168 \mathrm{eV}$ ) of a single negatively charged CdSe/ZnSe QD versus photon energy of the excitation in the range of excess energies between 0.08 and $0.14 \mathrm{eV}$ with respect to $E_{\mathrm{X}^{-}}$. The average excitation power is kept constant at $P_{\mathrm{exc}}=2 \mu \mathrm{W}$. Three main bright features (color-filled areas) at relative energies of $0.088,0.11$, and $0.13 \mathrm{eV}$ are identified with the $D s$ and the $P p$ triplet and singlet transitions. Colors match arrows in (a). Top: measurement, bottom: simulation. (c) Photoluminescence spectra of the same QD excited on the $P p$ triplet (green line) and the $D s$ (orange dashed line) resonances with $P_{\mathrm{exc}}=10 \mu \mathrm{W}$. Emission from the $\mathrm{X}^{-}$resonance and two biexcitonic transitions at $E_{\mathrm{XX}_{1}^{-}}=2.161 \mathrm{eV}$ and $E_{\mathrm{XX}_{2}^{-}}=2.155 \mathrm{eV}$ are observed for $P p$ triplet excitation. Biexcitonic emission is suppressed for $D s$ pumping. The inset depicts the polarization-resolved intensity of the PL maxima. Measurements are performed at a temperature of $T_{L}=2 \mathrm{~K}$.

exceeds the radiative lifetime of $500 \mathrm{ps}$ the sample needs for full electronic recombination. Therefore $T\left(P_{\mathrm{exc}}, E_{\mathrm{ph}}, t_{D}+t_{M}\right)$ effectively corresponds to the transmission of the sample in its ground state. By alternating between both settings in a quasi-lock-in scheme operating at a modulation frequency of $30 \mathrm{~Hz}$, we can measure $\Delta T / T$ in the sense of Eq. (2), under conditions referenced to the ground-state optical properties and without modulating the average excitation power.

\section{ENERGETIC STRUCTURE OF CdSe/ZnSe QUANTUM DOTS}

Understanding the ultrafast dynamics of few-fermion states in single QDs necessitates precise knowledge of their electronic level structure. To this end, we perform a detailed experimental and theoretical characterization of the emission and absorption properties of our systems. Figure 2(a) sketches the energetic spectrum of negatively charged QD eigenstates containing up to five fermionic quasiparticles. The panel is subdivided into three main columns where complexity is increasing from left to right due to the inclusion of manybody interactions. The left column depicts the energy scheme including the electron-electron exchange interaction. For the center column, the electron-hole exchange interaction has been considered, neglecting those parts of the interaction that would mix states with different spin configurations. Interactions that mediate those mixtures are included in the third column. Note that spin mixing due to the electron-hole interaction is typically associated with a violation of the cylindrical symmetry of the QD. At the start, we focus on the unmixed, well defined spin states (left and center column). In the following, the quantization axis is taken to be the $z$ axis. It is determined by the direction of pump and probe beams, coinciding with the growth direction of the samples and the external magnetic field. The orbital envelope wave functions in the valence band are denoted by capital letters and their conduction-band counterparts by lower-case labels, respectively. The ground state of the QDs we consider is given by a single electron in the $s$ shell with a spin projection of $\pm 1 / 2$, i.e., $1 s_{1 / 2}$. The lowest excited state consists of three charge carriers in their respective $s$ shells: two electrons with spin projections of $s_{i z}= \pm 1 / 2(i=1,2)$ and a heavy hole with an angular momentum projection of $j_{z}=$ $\pm 3 / 2$ are forming a $1 S_{3 / 2} 2 s_{1 / 2}$ configuration which represents the trion ground state (XGS). As the electron spins constitute a spin singlet $(S=0)$, the total angular momentum projection of the ground-state trion $F_{z}=s_{1 z}+s_{2 z}+j_{z}= \pm 3 / 2$ is defined by the heavy hole. In contrast to uncharged QDs, exciton ground states with $F_{z}= \pm 2$ (dark excitons), leading to dipole forbidden transitions and slow relaxation dynamics [49], are absent in this system. The radiative recombination from the 
XGS to the QD ground state is referred to as the fundamental trion transition $\mathrm{X}^{-}$[downward black arrow in Fig. 2(a)]. For increasing energy, we find a transition into the $1 D_{3 / 2} 2 s_{1 / 2}$ state (orange dashed arrow) [43]. Here, an electron and a hole are excited in the $s$ and $D$ shells, respectively, and the electron spins form a spin singlet. The relaxation of this state into the XGS is of particular interest to this work as the process requires minimum dissipation of energy, limited entirely by the intraband scattering of the hole. States with a $1 P_{3 / 2} 1 s_{1 / 2} 1 p_{1 / 2}$ configuration are higher in energy and characterized by a rich fine structure [11,50-52]. As the electrons now occupy different shells, the formation of both spin singlet $(S=0)$ as well as spin triplet states with an overall electron spin of $S=1$ is possible. To keep track of the nomenclature, it is convenient to represent these states in the form $\left|F_{z}\right\rangle_{S}=$ $\left|S^{2}, S_{z}\right\rangle\left|j_{z}\right\rangle$, where $S^{2}$ is the square of the overall electron spin. The subscript $S$ is added here to distinguish between states involving triplet and singlet electron spin configurations but with otherwise identical total angular momentum. As an example, the three-particle states involving electrons in a singlet configuration can be written as $| \pm 3 / 2\rangle_{0}=|0,0\rangle| \pm 3 / 2\rangle$, similarly for the states involving the electrons in the triplet configuration: $| \pm 1 / 2\rangle_{1}=|1, \mp 1\rangle| \pm 3 / 2\rangle,| \pm 3 / 2\rangle_{1}=|1,0\rangle| \pm 3 / 2\rangle$, and $| \pm 5 / 2\rangle_{1}=|1, \pm 1\rangle| \pm 3 / 2\rangle$.

The electron-electron (EEX) and electron-hole (EHX) exchange interactions modify the energetic structure of these states. The EEX lifts the degeneracy between singlet states with $S=0$ and triplet states with $S=1$ [53]. Here, the singlet states form the energetically higher states, that are separated from the triplet states by an energy $\Delta_{\mathrm{ee}}$. The energetic splitting of the $1 P_{3 / 2} 1 s_{1 / 2} 1 p_{1 / 2}$ singlet and triplet states is indicated with a solid black double arrow labelled $\Delta_{\text {ee }}$ in the left column of Fig. 2(a). Consideration of the EHX leads to an additional fine-structure in the $1 P_{3 / 2} 1 s_{1 / 2} 1 p_{1 / 2}$ triplet states, now consisting of nondegenerate $| \pm 1 / 2\rangle_{1},| \pm 3 / 2\rangle_{1}$, and $| \pm 5 / 2\rangle_{1}$ states [54], as shown in the center column of Fig. 2(a). The states are separated by the EHX interaction energy $\Delta_{\text {eh }}$, with $| \pm 1 / 2\rangle_{1}$ forming the energetically highest and $| \pm 5 / 2\rangle_{1}$ the lowest states. Transitions from the $1 s_{1 / 2}$ into the $1 P_{3 / 2} 1 s_{1 / 2} 1 p_{1 / 2}$ singlet (dark blue dot-dashed arrow) and the $| \pm 1 / 2\rangle_{1}$ and $| \pm 3 / 2\rangle_{1}$ triplet states (green arrows) are dipole allowed due to $\Delta F_{z}= \pm 1$ with respect to the QD ground state. Instead, excitation of the $| \pm 5 / 2\rangle_{1}$ triplet state requires $\Delta F_{z}= \pm 2$, which may be addressed optically only by two-photon absorption.

We now treat the case of mixed spin states [third column in Fig. 2 (a)]. There are two relevant mixings of spin configurations, indicated by dashed arrows in the right column of Fig. 2(a). On the one hand a combination of EEX and EHX as well as spin-orbit coupling [55] cause mixtures between the $| \pm 3 / 2\rangle_{1}$ trion triplet and the $| \pm 3 / 2\rangle_{0}$ singlet, denoted by $\delta_{\mathrm{ee}}$. On the other hand, a combination of EHX and valenceband mixing as well as higher terms of EHX [51] lead to mixtures between $|\mp 1 / 2\rangle_{1}$ and $| \pm 3 / 2\rangle_{1}$ triplet states, labeled by $\delta_{\text {eh }}$. Energy shifts caused by $\delta_{\text {ee }}$ and $\delta_{\text {eh }}$ are negligible. The mixtures modify the spin eigenstates. Considering the mixing between the triplet states, we define $\delta_{\mathrm{eh}} / \Delta_{\mathrm{eh}}=\alpha$ and the normalization factor $\eta=1 / \sqrt{1+\alpha^{2}}$. Thereby the triplet eigenstates can be written as superpositions of the pure triplet wave functions: $|X\rangle=\eta\left(| \pm 1 / 2\rangle_{1}+\alpha|\mp 3 / 2\rangle_{1}\right)$ and $|Y\rangle=$ $\eta\left(| \pm 3 / 2\rangle_{1}-\alpha|\mp 1 / 2\rangle_{1}\right)[11]$. Here, we assume the symmetry axes of the QD to be aligned to the $\mathrm{x}$ and $\mathrm{y}$ axes, respectively. Analogously, the mixing of the $F_{z}= \pm 3 / 2$ trion triplet and singlet states result in $|\Phi\rangle=\rho\left(| \pm 3 / 2\rangle_{1}+\varphi| \pm 3 / 2\rangle_{0}\right)$ [55]. Here, $\varphi=\delta_{\mathrm{ee}} / \Delta_{\mathrm{ee}}$ and $\rho=1 / \sqrt{1+\varphi^{2}}$.

The last transition that is relevant for this work is a cascaded two-photon absorption exciting a charged biexciton $\left(\mathrm{XX}^{-}\right)$, i.e., a five-particle state with $2 P_{3 / 2} 1 s_{1 / 2} 2 p_{1 / 2}$ configuration. The total angular momentum projection is $F_{z}= \pm 1 / 2$ due to the cancelation of contributions of both $P$-shell holes and $p$-shell electrons. Relaxation of the charged biexciton state initially involves scattering of the $P$-shell holes and one $p$-shell electron to their respective $s$ shells. This situation is indicated in the rightmost part of Fig. 2(a) with a slightly lowered biexciton ground-state energy level labelled $2 S_{3 / 2} 2 s_{1 / 2} 1 p_{1 / 2}$. The charged biexciton can relax via one-photon emission into the $1 S_{3 / 2} 1 s_{1 / 2} 1 p_{1 / 2}$ trion triplet states with respective angular momentum projections of $F_{z}= \pm 1 / 2$ or $\pm 3 / 2$, depending on the initial spin of the resident electron. Two emission lines [XX ${ }^{-}$, light blue and $\mathrm{XX}_{2}{ }^{-}$, red in Fig. 2(a)] are therefore expected [56] owing to the lifted degeneracy of the triplet states. It has to be noted that the final triplet states of this process are redshifted in energy with respect to the excited $1 P_{3 / 2} 1 s_{1 / 2} 1 p_{1 / 2}$ triplet states due to the relaxed $S$-shell hole. For clarity, only the two final states that occur in our experiments are depicted in Fig. 2(a). The full energetic spectrum of these excitations matches the structure of the $1 P_{3 / 2} 1 s_{1 / 2} 1 p_{1 / 2}$ states.

The optical transitions described above can now be identified experimentally by photoluminescence (PL) and photoluminescence excitation (PLE) spectroscopy. To this end, we decrease the bandwidth of our pump pulses to $0.3 \mathrm{meV}$ by using a grating-based 4-f pulse shaper. We then scan the excitation energy through the energy range from +0.07 to $+0.15 \mathrm{eV}$ with respect to the fundamental trion transition $E_{X^{-}}$, while at the same time detecting the PL emitted on the $\mathrm{X}^{-}$resonance. This procedure enables us to identify the frequencies where the system may be excited resonantly. The upper panel of Fig. 2(b) shows the PLE spectrum of a typical QD. The data have been taken at a temperature $T_{L}$ of $2 \mathrm{~K}$ and an average excitation power of $P_{\mathrm{exc}}=2 \mu \mathrm{W}$. The PLE spectrum shows three main absorption features at an energy detuning of $E-E_{X^{-}}=0.088$ (orange), 0.107 (green), and 0.130 (dark blue) eV, respectively. Especially the absorption line at $E-E_{X^{-}}=0.107 \mathrm{eV}$ displays a pronounced fine structure. To understand the relaxation mechanisms, it is crucial to link the energetic structure depicted in Fig. 2(a) to the measured PLE spectrum by an unambiguous identification of the observed absorption resonances. To this end, we implemented an extensive theoretical model of the energetic structure of $\mathrm{CdSe} / \mathrm{ZnSe}$ QDs. Based on the envelope function approximation, we consider the effective mass energies and include the direct and short-range exchange Coulomb interactions within a configuration interaction approach.

In the calculation, the heavy hole as well as the lowest conduction band are considered with a band gap of $1840 \mathrm{meV}$ [57,58], effective hole masses of $m_{h}^{x}=m_{h}^{y}=0.38 m_{0}$ and $m_{h}^{z}=m_{0}$ [57], and an effective electron mass of $m_{e}=0.13 m_{0}$ [58], with $m_{0}$ being the free-space electron mass. The QD 
confinement is approximated by a three-dimensional harmonic potential determined by the confinement lengths $L_{e}^{i}(i=x, y, z)$ of the electrons. Best agreement with the PLE data is achieved for a QD with $L_{e}^{x}=5.33 \mathrm{~nm}, L_{e}^{y}=4.4 \mathrm{~nm}$, and $L_{e}^{z}=2.262 \mathrm{~nm}$, suggesting a flat and slightly elongated shape $[45,59]$. Note that the confinement length is defined as 1/e of the ground-state probability density. Differences in confinement lengths between holes and electrons are described by the ratio $\beta=L_{h}^{i} / L_{e}^{i}=1.3$, in qualitative agreement with previous work [43]. The holes and electrons are expanded in Cartesian eigenfunctions of the harmonic confinement up to quantum numbers $\left(a_{x}^{h}=5, a_{y}^{h}=5, a_{z}^{h}=3\right)$ and $\left(a_{x}^{e}=3, a_{y}^{e}=3, a_{z}^{e}=1\right)$, respectively, and about 12000 combinations of trion states are considered to achieve sufficient convergence. For the treatment of Coulomb interaction we follow references $[60,61]$. While often the direct Coulomb interaction is assumed to be screened by the static dielectric constant of the bulk material $\epsilon_{r}^{\text {bulk }}=9.2$ [58], other studies [58,62-64] indicate a reduction of $\epsilon_{r}$ in QDs, e.g., due to a decrease of phononic screening. Here, we use $\epsilon_{r}=7.4$ to fit the experimental data. We take into account the shortrange Coulomb exchange interaction via the coupling strength $E_{x}^{s} V_{\text {cell }}=576 \mathrm{meV} \mathrm{nm}^{3}, V_{\text {cell }}$ being the volume of the unit cell. In calculations for neutral QDs, this value results in a Stokes shift that is in good agreement with the well-established findings in Ref. [65].

The calculated absorption spectrum is shown in the bottom panel of Fig. 2(b). Quantitative agreement with the measured PLE spectrum in the upper panel of Fig. 2(b) is achieved, leading to an assignment of all relevant transitions: the feature at $0.088 \mathrm{eV}$ is identified as the transition from the QD ground state to the $1 D_{3 / 2} 2 s_{1 / 2}$ state. For abbreviation, we will refer to this resonance as the $D s$ transition. It appears to consist of two maxima that are not completely resolved. The main feature at $0.107 \mathrm{eV}$ corresponds to the formation of the $1 P_{3 / 2} 1 s_{1 / 2} 1 p_{1 / 2}$ state in a triplet spin configuration, in the following referred to as the $P p$ triplet transition. Here we clearly resolve the splitting of the transition into two main lines at $E-E_{X^{-}}=$ 0.105 and $0.108 \mathrm{eV}$ as well as two minor spectral features on the low-energy side and an additional weak absorption on the high-energy tail. We ascribe the two main lines to an absorption into $| \pm 1 / 2\rangle_{1}$ and $| \pm 3 / 2\rangle_{1}$ configurations. The weak maxima on both sides of the main resonances are most likely due to admixtures of the discussed bright states to nominally dark states [43]. The overall asymmetry of the absorption feature with a pronounced extension toward higher energies is attributed to acoustic phonon-assisted absorption. At a relative energy of $E-E_{X^{-}}=0.130 \mathrm{eV}$, we observe another distinct resonance which is associated with the absorption into the $1 P_{3 / 2} 1 s_{1 / 2} 1 p_{1 / 2}$ singlet state, i.e., the $P p$ singlet resonance. The measured energetic splitting of respective singlet and triplet states is consistent with previous work [66]. The $P p$ triplet transition offers the highest oscillator strength, rendering it interesting for experiments requiring maximally efficient initialization of the quantum emitter. If we compare the excitation energies of the $1 P_{3 / 2} 1 s_{1 / 2} 1 p_{1 / 2}$ singlet and $1 D_{3 / 2} 2 s_{1 / 2}$ states, it is evident that relaxation of the $1 P_{3 / 2} 1 s_{1 / 2} 1 p_{1 / 2}$ singlet to the $\mathrm{XGS}$ requires dissipation of an additional excess energy of $42 \mathrm{meV}$.

To complete the characterization of the quantum system and to further support the assignments done so far, we have also conducted energy- and polarization-resolved PL emission measurements. Figure 2(c) shows the spectrally resolved normalized PL intensity at a temperature $T_{L}$ of $10 \mathrm{~K}$ and at a relatively high excitation with $P_{\mathrm{exc}}=10 \mu \mathrm{W}$. The PL signal is compared when the QD is excited on the $D s$ resonance (orange dashed line) and the $P p$ triplet resonance (green line). Both spectra are normalized to the intensity of the fundamental trion transition $\mathrm{X}^{-}$. The $\mathrm{X}^{-}$resonance at $E_{X^{-}}=2.168 \mathrm{eV}$ is the most intense feature in the PL emission. The asymmetric line shape results from acoustic phonons forming a distinctive tail on the low-energy side of the emission. Two additional features labelled $\mathrm{XX}_{1}{ }^{-}$and $\mathrm{XX}_{2}{ }^{-}$can be detected, which are redshifted by 7 and $13 \mathrm{meV}$, respectively. Interestingly, these lines are only present when excitation is tuned to the $P p$ triplet resonance. We assign these lines to the recombination of charged biexcitons. A quadratic dependence of the emission intensity on the excitation power together with the double-line feature are all characteristics of this higher-order process. This assignment is also consistent with the fact that excitation on the $D s$ resonance, resulting in a full occupation of the electron $s$ shell after absorption of one photon, leads to blocking of the formation channel for a biexciton. The $P p$ resonances impose no such limitations, in full accordance with our data. In addition, we have studied the polarization characteristics of the $\mathrm{X}^{-}$and $\mathrm{XX}^{-}$emission. The inset of Fig. 2(c) depicts the spectrally integrated intensity of the $\mathrm{X}^{-}$(black), $\mathrm{XX}_{1}^{-}$(light blue), and $\mathrm{XX}_{2}^{-}$(red) emission lines versus the polarization angle, respectively, as analyzed in front of the entrance slit of the spectrometer and corrected for the monochromator grating response. We observe a nearly unpolarized emission of the $\mathrm{X}^{-}$resonance and mutually orthogonal, elliptical polarization of the biexciton recombination lines. As mentioned above, the trion ground state is doubly degenerate in the absence of a magnetic field, leading to the unpolarized emission we observe. The doubly degenerate biexciton states relax into the $| \pm 1 / 2\rangle_{1}$ and $| \pm 3 / 2\rangle_{1}$ configurations. Under consideration of spin mixing, these are no longer pure states but instead possess the admixed character of $|X\rangle$ and $|Y\rangle$ explained above. The recombination thus results in the emission of elliptically polarized light with mutually perpendicular axes. The degree of polarization depends on the magnitude of the coupling constant $\alpha=\delta_{\text {eh }} / \Delta_{\text {eh }}$. These findings provide further support for our level assignments. Consequently, we have developed a self-consistent picture of the multitude of few-particle states and optical transitions in our singly charged CdSe/ZnSe QDs.

\section{ULTRAFAST INTRABAND RELAXATION OF THE $1 D_{3 / 2} 2 s_{1 / 2}$ AND $1 P_{3 / 2} 1 p_{1 / 2} 1 s_{1 / 2}$ STATES}

To gain insight into the few-fermion relaxation processes that govern the dynamical behavior of our single-photon amplifier, we now perform pump-probe measurements with femtosecond time resolution. To this end, we spectrally tune the pump pulses to one of the identified absorption resonances and center the probe spectrum at the $\mathrm{X}^{-}$transition. Pump and probe fields are set to linear polarization, thus enabling excitation and readout of all dipole-allowed transitions. The bandwidth of the 100-fs probe pulses enables us to detect signals in an energy interval of $10 \mathrm{meV}$ around the $\mathrm{X}^{-}$resonance with nearly frequency-independent signal-to-noise ratio. Typical average 
(a)

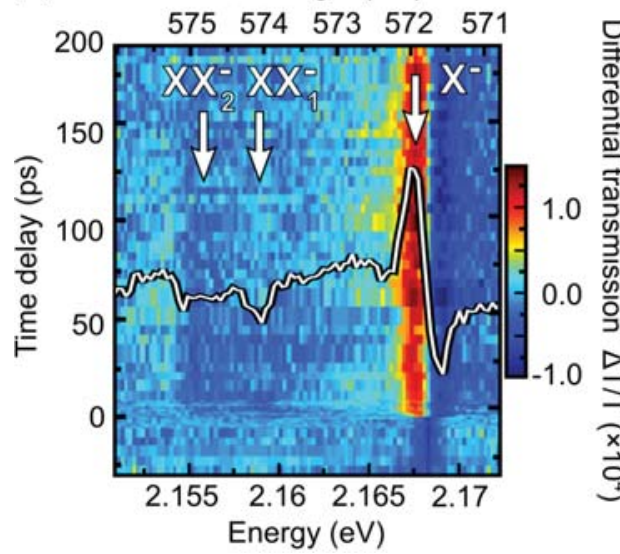

(b)

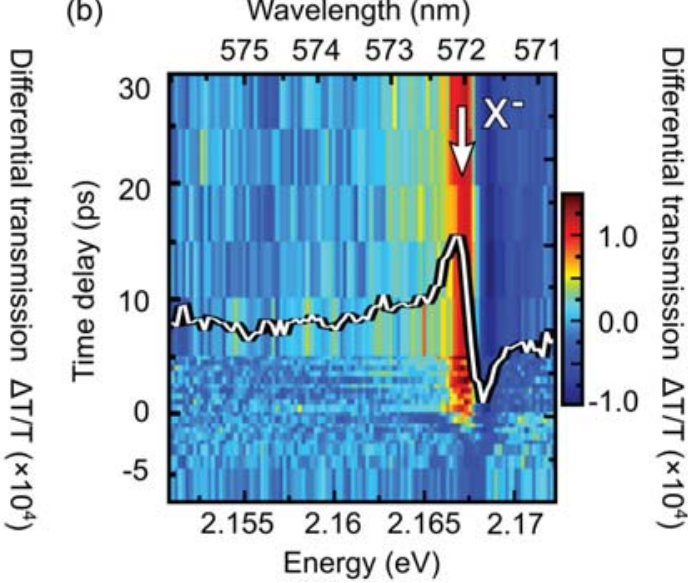

FIG. 3. (a) Spectrally resolved differential transmission $\Delta T / T$ of a single CdSe/ZnSe QD for time delays $t_{D}$ between pump and probe pulses from -30 to $+200 \mathrm{ps}$ at $T_{L}=10 \mathrm{~K}$. The photon energy of the pump pulses is centered on the $P p$ triplet resonance $\left(P_{\text {exc }}=10 \mu \mathrm{W}\right)$. The probe pulses are tuned to the $\mathrm{X}^{-}$resonance $\left(P_{\mathrm{pro}}=2 \mu \mathrm{W}\right)$. The spectral positions of the fundamental trion resonance $\left(\mathrm{X}^{-}\right)$and biexciton resonances $\left(\mathrm{XX}_{1}^{-}\right.$and $\left.\mathrm{XX}_{2}^{-}\right)$are indicated by white arrows. A differential transmission spectrum averaging over the time interval from +5 to $+55 \mathrm{ps}$ is shown as a white line. (b) Spectrally resolved differential transmission of the same QD for excitation of the $D s$ transition and time delays from -7.5 to $+30 \mathrm{ps}$. The white line depicts the average of the spectrally resolved differential transmission for temporal delays between +5 and $+30 \mathrm{ps}$.

powers for incident excitation and readout pulse trains are 10 (corresponding to a pulse area of $0.8 \pi$ ) and 2 (area of 0.2 $\pi) \mu \mathrm{W}$, respectively. The samples are cooled to a substrate temperature of $T_{L}=2 \mathrm{~K}$.

Figure 3(a) shows the color-coded spectrally and temporally resolved differential transmission signal $\Delta T / T$ of a single QD when the central photon energy for excitation is set to $E_{\text {exc }}=2.275 \mathrm{eV}$. Under those conditions, both of the closely spaced $| \pm 3 / 2\rangle_{1}$ and $| \pm 1 / 2\rangle_{1} P p$ triplet resonances are located within the bandwidth of the 600 -fs pump pulse. A clear effect is visible at the spectral position of the $\mathrm{X}^{-}$transition (white arrow labelled $\mathrm{X}^{-}$). For small negative time delays, where probe pulses preceed the pump, negative $\Delta T / T$ is caused by a perturbed free induction decay $[7,21]$. A sharp onset of positive differential transmission occurs at the fundamental trion resonance around $t_{D}=0 \mathrm{ps}$. It is followed by an additional increase on a time scale of approximately $50 \mathrm{ps}$. At positive delay times, there are two fundamental processes that lead to differential transmission signals in these systems [7,43]: (i) the Coulomb interaction with photoexcited carriers may shift absorption resonances quasi-instantaneously. (ii) Finite carrier occupation of the states first causes bleaching due to Pauli blocking and might eventually lead to single-photon gain when inversion is achieved. In case of pumping the $P p$ transitions, the onset of the latter effect is delayed due to the retarded intraband relaxation of the photogenerated electron and hole. In the following, we will single out the temporal dynamics of $\mathrm{X}^{-}$occupation from the differential transmission traces.

On the low-energy side of the main dynamic feature at $\mathrm{X}^{-}$, weak negative differential transmission signals are discernible in Fig. 3(a), coinciding with the energies of the biexcitonic emission lines $\mathrm{XX}_{1}^{-}$and $\mathrm{XX}_{2}{ }^{-}$. These features are a consequence of an excitation of charged biexciton states. The excited-state absorption becomes possible due to the creation of electron-hole pairs in their respective $p$ shells. As discussed in the context of Fig. 2(a), those transitions are redshifted with respect to the $\mathrm{X}^{-}$line due to $\mathrm{EHX}$ and Coulomb interactions inherent to the trion triplet states. The onset of the induced $\mathrm{XX}^{-}$ absorption signals is immediate. Interestingly, the biexcitonic features seem to decay on approximately the same 50-ps time scale, which also characterizes the additional increase of $\Delta T / T$ at the $\mathrm{X}^{-}$resonance. This finding may be related to the intraband relaxation of the excited $p$-shell electron into the $s$ shell, thereby closing the biexcitonic absorption channel.

Next, we turn our attention to the dynamics following the $D s$ transition which is excited by pumping at $E_{\text {exc }}=$ $2.256 \mathrm{eV}$. Figure 3(b) depicts the differential transmission versus probe photon energy for time delays between -8 and $+30 \mathrm{ps}$. At negative $t_{D}$, spectral oscillations characteristic for the perturbed dephasing of the probe-induced polarization are clearly visible in analogy to excitation of the $P p$ triplet resonance. For positive time delays, a striking feature is the absence of the transient biexcitonic absorption signatures, in full agreement with the missing biexcitonic luminescence seen in Fig. 2(c). Now the excitation of an electron-hole pair in their respective s and $D$ shells leads to an instantaneous and full occupation of the electron $s$ shell. Consequently, it is not possible to absorb a probe photon on the $\mathrm{XX}^{-}$transition, in accord with our assignment of the electronic structure associated with the absorption resonances. Furthermore, we observe that the ultrafast dynamics following $D s$ initialization is qualitatively different from that of the $P p$ excitation: the differential transmission in Fig. 3(b) rises to a global maximum at a much faster rate, consistent with the fact that it is only the intraband scattering of the hole that dictates the time scale for the relaxation of the three-fermion system into its ground state. These findings already indicate the possibility to readily single out the differences in the relaxation of various nonequilibrium electronic configurations of a single QD. 
(a)

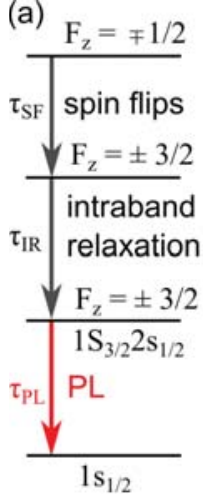

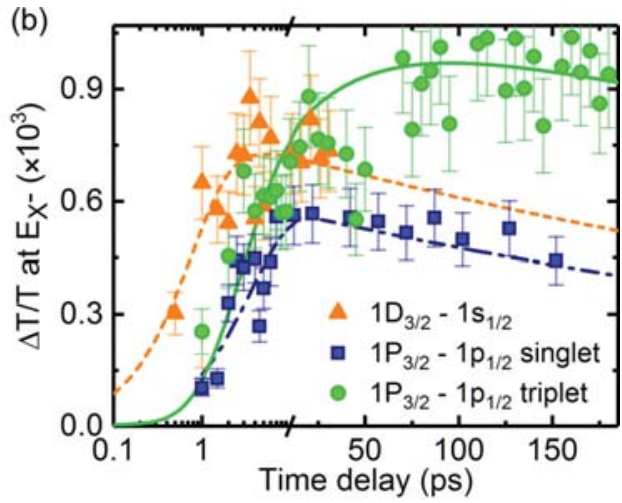

FIG. 4. (a) Schematic of a four-level-scheme to model onset and decay times of the observed $\Delta T / T$ signatures. The signal on the $\mathrm{X}^{-}$ resonance is proportional to the occupation of the $1 S_{3 / 2} 2 s_{1 / 2}$ state. For relaxation of excited trion states with $F_{z}= \pm 3 / 2$ only the lower three levels are considered. The complete four-level-model is used to extract the dynamics following the excitation of the $F_{z}= \pm 1 / 2$ triplet state. (b) Comparison of the temporal evolution of differential transmission signals measured at the $\mathrm{X}^{-}$transition when the single $\mathrm{QD}$ is resonantly excited on the $D s$ (orange triangles, dashed line), $P p$ triplet (green circles, solid line), and $P p$ singlet (dark blue squares, dot-dashed line) resonances. Lines correspond to least-squares fits using the models outlined in (a). Data are obtained at $T_{L}=10 \mathrm{~K}$.

We now focus on the transient signals at the $\mathrm{X}^{-}$resonance. To analyze the dynamics of our observable, namely the differential transmission of the trion ground state, we introduce a rate-equation model based on a four-level manifold. A sketch detailing this structure is shown in Fig. 4(a). The uppermost level is reserved for the states with $F_{z}= \pm 1 / 2$ which relax toward the states with $F_{z}= \pm 3 / 2$ at a transition rate of $1 / \tau_{\mathrm{SF}}$. This channel is introduced to account for the intraband scattering processes which can involve electron and hole spin flips. A second relaxation step from excited trion configurations with $F_{z}= \pm 3 / 2$ to the trion ground state $\left(1 S_{3 / 2} 2 s_{1 / 2}\right)$ is considered with a characteristic relaxation time $\tau_{\mathrm{IR}}$. This interval accounts for scattering processes that require only energy dissipation $\left(\Delta F_{z}=0\right)$, as mediated, e.g., by acoustic or optical phonons $[36,67]$. Finally, the last transition with a decay time of $\tau_{\mathrm{PL}}$ accounts for the spontaneous recombination of the trion ground state towards the QD ground state $1 s_{1 / 2}$. This population decay exponentially diminishes the occupation of the XGS, leading to a decrease in the gain signal for long time delays. The interband recombination time $\tau_{\mathrm{PL}}$ is determined to be (500 \pm 50) ps using an avalanche photodiode in time-correlated singlephoton counting measurements. Time-dependent solutions of the model are convolved with the measured temporal crosscorrelation of the laser pulses, which together with $\tau_{\mathrm{PL}}$ are used as input parameters to the model calculation. In case of $P p$ excitation, a bleaching component of the $\mathrm{X}^{-}$absorption arises instantaneously, i.e., with the time-integrated cross-correlation between pump and probe. This process is caused by the Coulomb renormalization [7] replacing the $\mathrm{X}^{-}$resonance by the biexcitonic $\mathrm{XX}^{-}$absorption lines. When pumping the $D s$ transition, part of the differential transmission at $\mathrm{X}^{-}$occurs instantly as well, this time due to the Pauli blocking induced by direct excitation of the electron into the $s$ shell. Both phenomena decay due to spontaneous recombination of the trion ground state and are taken into account when extracting dynamical information from our data.

We are now ready to evaluate the differences in ultrafast dynamics upon excitation of the major dipole-allowed transitions. To enhance the signal-to-noise ratio, we spectrally integrate the positive part of the differential transmission signal around the position of the $\mathrm{X}^{-}$resonance. Figure 4(b) shows the dynamics resulting from excitation of the $1 D_{3 / 2} 2 s_{1 / 2}(D s$, orange triangles, dashed line), $1 P_{3 / 2} 1 s_{1 / 2} 1 p_{1 / 2}$ triplet $(P p$ triplet, green circles, solid line), and $1 P_{3 / 2} 1 s_{1 / 2} 1 p_{1 / 2}$ singlet ( $P p$ singlet, dark blue squares, dot-dashed line) resonances. Strong differences in the relaxation traces are immediately evident. The traces resulting from pumping of the $D s$ and the $P p$ singlet states show a fast temporal onset on a few-picosecond time scale followed by a monotonous decay. In contrast, when excitation is tuned to the $P p$ triplet transition, there exists an additional and pronounced increase of $\Delta T / T$ leading to a global maximum around $t_{D}=100$ ps. This difference may be understood considering the spin configurations of initial and final states. Scattering from $D s$ and $P p$ singlet states to the XGS necessitates only transitions conserving the spin projection, whereas relaxation from the $1 P_{3 / 2} 1 s_{1 / 2} 1 p_{1 / 2}$ triplet states requires also a change in the total electron spin. We now perform least-square fits for the cases with fast dynamics using the analytical solution of the four-level rate equation model in the limit of $\tau_{\mathrm{SF}} \rightarrow 0$, effectively considering a three-level system. For the situation of the $D s$ transition, we extract an onset time of differential transmission of $\tau_{h}=(780 \pm 380) \mathrm{fs}$. This ultrafast dynamics is solely determined by scattering of the $D$-shell heavy hole into the $S$ shell. Instead, the relaxation of the $1 P_{3 / 2} 1 s_{1 / 2} 1 p_{1 / 2}$ singlet state to the trion ground state exhibits a time constant of $\tau_{s}=(3.6 \pm 0.4)$ ps. Qualitatively, this longer time scale may be explained by the fact that here the intraband relaxation requires scattering of both the photoexcited electron and hole. A closer comparison of energies of the singlet lines in the PLE spectrum [Fig. 2(b)] reveals that in the case involving the $P p$ transition there is an additional excess energy of $42 \mathrm{meV}$ stored in the few-fermion system, in contrast to the $D s$ case. At an excess energy of $88 \mathrm{meV}$, the $D$ hole of the $1 D_{3 / 2} 2 s_{1 / 2}$ state has to scatter with at least three vibrational quanta [e.g., 2 longitudinal optical (LO) phonons and one acoustic mode] in order to relax toward the XGS. The energy of the zone-centered LO phonon is $30 \mathrm{meV}$ in $\mathrm{CdSe}$ [68]. In contrast, the $p$-shell electron and $P$-shell hole in the $1 P_{3 / 2} 1 s_{1 / 2} 1 p_{1 / 2}$ singlet have to dissipate 86 and $44 \mathrm{meV}$ of energy, which may be provided by at least three and two phonons, respectively. A fivefold increase in the intraband relaxation time observed for the $1 P_{3 / 2} 1 s_{1 / 2} 1 p_{1 / 2}$ state in comparison to the $1 D_{3 / 2} 2 s_{1 / 2}$ configuration can be directly attributed to the two additional scattering events between vibrational quanta and lighter fermions [19]. In contrast to the intraband dynamics of the singlets, the differential transmission trace for excitation on the $P p$ triplet transitions reveals a fundamentally different relaxation behavior. The dynamics includes two distinct time scales, namely, a fast onset of the signal near $t_{D}=0$ due to a combination of bleaching and rapid single photon gain and a retarded increase within multiple tens of picoseconds. It is only by consideration of the full model consisting of the formal description of the bleaching component, a reduced three-level 
manifold, and a four-level manifold that we are able to extract the fast time scale to be $\tau_{3 / 2}=(2.9 \pm 0.6) \mathrm{ps}$ and a risetime of the delayed component at $\tau_{1 / 2}=(55 \pm 10) \mathrm{ps}$. The maximum in $\Delta T / T$ at $t_{D}=100 \mathrm{ps}$ results from the interplay of the time constants $\tau_{1 / 2}$ and $\tau_{\mathrm{PL}}$.

To understand the origin of the two-time-scale relaxation process one has to consider that the two $1 P_{3 / 2} 1 s_{1 / 2} 1 p_{1 / 2}$ triplet states $| \pm 1 / 2\rangle_{1}$ and $| \pm 3 / 2\rangle_{1}$ are excited simultaneously. First, we discuss relaxation of the $| \pm 3 / 2\rangle_{1}$ triplet state to the XGS. The transition from the triplet $(S=1)$ to the singlet $(S=0)$ configuration necessitates that the total electron spin has to change by 1 . In the case of $| \pm 3 / 2\rangle_{1}$, however, no variation in the total spin projection is required. A microscopic picture of the spin relaxation process detected in our experiments is as follows: mixing of states due to the spin mixing part $\delta_{\text {ee }}$ of the EEX (see $|\Phi\rangle=\rho\left(| \pm 3 / 2\rangle_{1}+\varphi| \pm 3 / 2\rangle_{0}\right)$ in Sec. III and definitions therein) results in a finite overlap of $|\Phi\rangle$ with purely singlet-type wave functions $[53,55]$. $\delta_{\text {ee }}$ has been shown to take large values depending on the geometry of the QD, giving rise to a strong mixing of states and a direct relaxation channel for the nominally dark transition of the trion triplet states into the singlet-type trion ground state [55,69]. Applying Fermi's golden rule to the spin parts of the wave functions, we can estimate the relaxation time $\tau_{3 / 2}$ as a function of the relaxation time $\tau_{s}$ of the $1 P_{3 / 2} 1 p_{1 / 2} 1 s_{1 / 2}$ singlet states to be $\tau_{s}=\varphi^{2} \rho^{2} \tau_{3 / 2}$. As $\varphi^{2} \rho^{2}<1$, the triplet-to-singlet relaxation time is expected to take longer compared to a singlet-to-singlet transition at the same energy. The measured decay times show that relaxation of the $| \pm 3 / 2\rangle_{1}$ triplet states at $\tau_{3 / 2}=(2.9 \pm 0.6) \mathrm{ps}$ is in fact faster than the relaxation of the $1 P_{3 / 2} 1 s_{1 / 2} 1 p_{1 / 2}$ singlet with $\tau_{s}=(3.6 \pm 0.4) \mathrm{ps}$. This difference is attributed to the energetic landscape of the intraband scattering. Indeed, the PLE measurements confirm that the $| \pm 3 / 2\rangle_{1}$ to XGS transition is at a lower energy compared to that of the $1 P_{3 / 2} 1 s_{1 / 2} 1 p_{1 / 2}$ singlet. In agreement with the $D s$ and $P p$ singlet relaxation rates, this reduction in energy results in fewer phonon scattering events and a comparatively higher relaxation rate.

In contrast to the $| \pm 3 / 2\rangle_{1} \rightarrow| \pm 3 / 2\rangle_{0}$ transition, the relaxation of the $| \pm 1 / 2\rangle_{1}$ triplet states into the XGS has to involve changes both in the total electronic spin and its $z$ projection. For this we consider the mixing of $| \pm 3 / 2\rangle_{1}$ and $|\mp 1 / 2\rangle_{1}$ triplet states due to the spin mixing part $\delta_{\text {eh }}$ of the EHX, namely $|Y\rangle=\eta\left(| \pm 3 / 2\rangle_{1}-\alpha|\mp 1 / 2\rangle_{1}\right)$, as discussed in Sec. III. The complete relaxation path involves scattering from the $|\mp 1 / 2\rangle_{1}$ to the $| \pm 3 / 2\rangle_{1}$ configuration within the mixed triplet manifold and subsequent relaxation into the ground-state $1 S_{3 / 2} 2 s_{1 / 2}$ singlet via the $|\Phi\rangle$ admixture, as discussed above. If we adopt this point of view, it becomes clear that the measured long temporal component of the population decay $\tau_{1 / 2}$ is associated with the relatively weak strength of the admixture within the manifold of the triplet states. The relationship of $\tau_{1 / 2} \approx 19 \tau_{3 / 2}$ is consistent with this picture and indicates weak spin mixing due to EHX, where $\delta_{\text {eh }} \ll \Delta_{\text {eh }}$. In fact, from the estimate of the triplet-to-singlet relaxation via Fermi's golden rule, we can expect the long time scale to be given by $\tau_{3 / 2}=\eta^{2} \alpha^{2} \tau_{1 / 2}$ and hence $\delta_{\mathrm{eh}} / \Delta_{\mathrm{eh}} \approx \sqrt{\tau_{3 / 2} / \tau_{1 / 2}}=0.23 \pm 0.05$. Typical energetic separations of the $| \pm 3 / 2\rangle_{1}$ and $| \pm 1 / 2\rangle_{1}$ triplet states $\left(\Delta_{\mathrm{eh}}\right)$ have been determined to be in the range from 1 to $4 \mathrm{meV}$ via PLE measurements of various QDs. Instead, the spin mixing part of the EHX $\left(\delta_{\text {eh }}\right)$ can be directly read out from the splitting of the bright excitonic fine-structure states in electrically neutral QDs [54]. The existence of uncharged QDs in our sample lets us determine these values to be in the range of 0.5 to $3 \mathrm{meV}$. From these spectroscopic measurements, the ratio $\delta_{\mathrm{eh}} / \Delta_{\mathrm{eh}}$ is estimated to be in the range of 0.125 to 3 , in a good quantative agreement with the information extracted from the time-resolved traces. In our picture, the required change in the $z$ projection of the total angular momentum of the trion by $\Delta F_{z}= \pm 2$ (from $F_{z}=\mp 1 / 2$ to $\pm 3 / 2$ ) is provided by a simultaneous electron and hole spin flip process $[55,70,71]$. Alternatively, a competing relaxation from the $F_{z}= \pm 1 / 2$ to $\pm 3 / 2$ triplet states involving a flip of an individual electron spin via a cross-relaxation between the electronic and nuclear spin systems (nuclear Overhauser effect) is also possible. However, this process has been shown to be suppressed in zero-dimensional systems [55,70,72]. Furthermore, dephasing of the spin polarization in similar QDs has been determined to take up to several nanoseconds $[14,15,73]$. As a result, individual electron spin-flip processes can be neglected in the consideration of the ultrafast dynamics, thus allowing direct determination of the admixed characters of the electronic wavefunctions.

So far, we have analyzed the dynamics of three addressable excited trion states. In the remainder of the paper, we concentrate on two of these scenarios. In the first, the subpicosecond initialization of single-photon gain available after resonant excitation of the $1 D_{3 / 2} 2 s_{1 / 2}$ state is attractive for future applications in ultrafast quantum optics. With this in mind, in the next section, we investigate the hole relaxation process in different QDs and study the size-dependence for initialization of gain. In the second case, relaxation of the $1 P_{3 / 2} 1 s_{1 / 2} 1 p_{1 / 2}$ state occurs on a slower time scale but a larger dipole moment associated with the initialized transition promises a more efficient deterministic quantum amplifier. This scenario is studied in Sec. VI.

\section{ENERGY DEPENDENCE OF THE INTRABAND RELAXATION TIME OF THE EXCITED HOLES}

To further investigate the relaxation mechanisms and to explore the possibility of tuning the onset time of gain from the relaxation of the $1 D_{3 / 2} 2 s_{1 / 2}$ state, measurements of the corresponding relaxation time for four individual QDs are shown in Fig. 5. The nanocrystals are selected according to the energetic difference $E_{D s}-\mathrm{E}_{\mathrm{X}^{-}}$between the fundamental trion transition $\mathrm{X}^{-}$and the $D$ s resonance, as determined from individual PLE measurements (not shown). This energy corresponds to the relaxation of the $D$-shell hole into the respective $S$ shell. $E_{D s}-\mathrm{E}_{\mathrm{X}^{-}}$is specific to the spatial dimensions of a QD. It varies throughout the ensemble due to the underlying size distribution. By studying ultrafast dynamics in QDs of different size, it is thus possible to map out the functional dependence of the relaxation time on the amount of excess energy dissipated by the hole in its transition to the XGS. To this end, individual QDs with energy differences $E_{D_{s}}-E_{X^{-}}$between 79 and $100 \mathrm{meV}$ are selected. The relaxation times are obtained from the three-level model fits of the time-resolved normalized differential transmission traces at the spectral position of the 


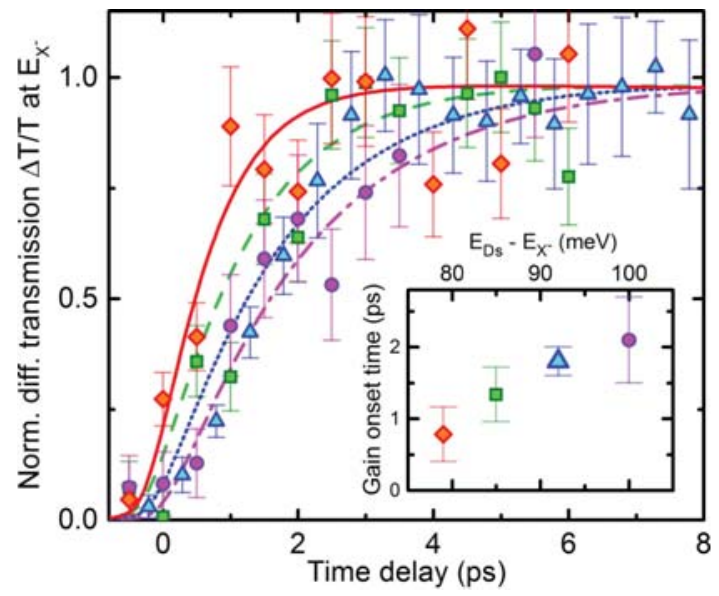

FIG. 5. Ultrafast relaxation of the $D$-shell hole in four QDs of varying size when excited on the respective $D s$ transitions. The energy splitting of the $1 D_{3 / 2} 2 s_{1 / 2}$ and $1 s_{1 / 2}$ states $E_{D s}-E_{X^{-}}$is 79 (red diamonds, solid line), 85 (green squares, dashed line), 92 (blue triangles, dotted line), and 100 (purple circles, dot-dashed line) meV, respectively. The main panel shows the onset of the differential transmission signal on the $\mathrm{X}^{-}$resonance and corresponding leastsquares fits as a function of time delay $t_{D}$ from -0.5 to $+8 \mathrm{ps}$. The inset depicts the extracted times constants in relation to the measured energy difference $E_{D s}-E_{X^{-}}$for the investigated QDs. Data are obtained at a temperature of $T_{L}=10 \mathrm{~K}$.

$\mathrm{X}^{-}$resonance when QDs are excited on the respective $D s$ transitions (Fig. 5). We find relaxation times of (780 \pm 380$) \mathrm{fs}$ for $E_{D s}-E_{X^{-}}=79 \mathrm{meV}$ (orange diamonds and red solid line), $(1.3 \pm 0.4)$ ps for $E_{D s}-E_{X^{-}}=85 \mathrm{meV}$ (green squares and dashed line), $(1.8 \pm 0.2)$ ps for $E_{D s}-E_{X^{-}}=92 \mathrm{meV}$ (blue triangles and dotted line), and $(2.1 \pm 0.6)$ ps for $E_{D s}-$ $E_{X^{-}}=100 \mathrm{meV}$ (purple dots and dash-dotted line). The inset of Fig. 5 depicts the extracted onset time of gain in relation to the probed $E_{D s}-E_{X^{-}}$detuning, clearly showing a factor of 3 increase in the scattering rate for a $20 \%$ reduction in the hole excess energy from 100 to $79 \mathrm{meV}$. While a microscopic description of hole intraband relaxation is beyond the scope of this work, the dependence observed in Fig. 5 qualitatively fits a perturbative picture based on interaction with multiple phonons. The scattering rate shows inverse scaling with the dissipated energy, which is distributed between the optical and acoustic vibrational quanta according to the eigenfrequencies and momentum conservation rules in the nanoconfined system. It is interesting to point out that the energy range investigated here captures a transition from (i) hole relaxation via $2 \mathrm{LO}$ phonons and high energetic acoustic phonons to (ii) relaxation via 3 LO phonons and acoustic phonons. Due to the larger mismatch in momentum for higher acoustic phonon energies and a lower scattering rate in comparison to LO phonon energy, we expect to observe resonances in the spectrum of relaxation times at energies corresponding to an integer multiple of LO phonons. Such features could potentially be investigated in future studies. Nevertheless, a promising route for speeding up the initialization of single-photon gain in single QDs is apparent: for instance, by reducing the energy detuning below $60 \mathrm{meV}$ (reducing the number of the involved LO vibrational quanta) it might be possible to increase the operating bandwidth of quantum amplifiers into the few-terahertz range.

\section{EXTERNAL MAGNETIC FIELD AND EXTRACTION OF MAXIMAL SINGLE-PHOTON GAIN}

Finally, we turn to the initialization procedure of singlephoton gain by means of the low-energy triplet configurations. Despite the longer intraband scattering times, this scenario is advantageous for enhancing the efficiency of quantum amplifiers due to large dipole moments associated with these transitions. Additionally, the lower excitation power required to invert the quantum system minimizes any parasitic excitation processes and potential heat input, thus ensuring highest fidelity for the opration of the quantum amplifier. In the ideal case, the normalized differential transmission signal corresponding to single-photon gain should be twice the value of the bleaching plateau $[43,74]$. As evident from the observations in Sec. IV, it is not possible to directly extract full gain from the combined relaxation of the $| \pm 3 / 2\rangle_{1}$ and $|\mp 1 / 2\rangle_{1}$ triplet states. As a result of the degeneracy in the QD ground state and the finite Overhauser coupling to the nuclear spin bath, the resident electron experiences stochastic fluctuations in the spin projection. Furthermore, the energy splitting between the $| \pm 3 / 2\rangle_{1}$ and $| \pm 1 / 2\rangle_{1}$ states of only a few meV makes it difficult to initialize them individually by an ultrafast and therefore broadband excitation pulse. These challenges can be addressed by application of an external magnetic field in Faraday geometry, where lifting of the double degeneracy of the QD ground-state results in a deterministic alignment of the resident electron spin. At a field magnitude of $5 \mathrm{~T}$ and a temperature of $1.6 \mathrm{~K}$, a Zeeman splitting of $0.28 \mathrm{meV}$ in the ground state of our QDs ensures nearly 70 percent occupation of the lower-energy electron state with $s_{z}=-1 / 2$. It also becomes possible to selectively initialize components of the triplet-to-singlet transitions with $\sigma^{-}$or $\sigma^{+}$circularly polarized photons, thereby addressing either the $|-3 / 2\rangle_{1}$ or the $|+1 / 2\rangle_{1}$ states, respectively.

The main panel of Fig. 6 shows the temporal traces of the differential transmission for excitation of the $|-3 / 2\rangle_{1}$ (blue rectangles) and the $|+1 / 2\rangle_{1}$ (red circles) triplet states for time delays between -10 and $+130 \mathrm{ps}$. The pump is switched between the $\sigma^{-}$or $\sigma^{+}$orientations and the probe is kept at $\sigma^{-}$polarization, on resonance with the transition from the XGS to the QD ground state with a single electron of $s_{z}=-1 / 2$. After the excitation of the $|+1 / 2\rangle_{1}$ triplet state, we observe an initially fast increase in the signal to a value of approximately $\Delta T / T=0.48 \times 10^{-3}$ (lower orange-shaded region). We ascribe this quasi-instantaneous step in the signal to the process of ultrafast bleaching of the $\mathrm{X}^{-}$resonance due to the large level shift via Coulomb renormalization. Owing to its retarded character, the additional increase in differential transmission has to be caused by a finite $\mathrm{X}^{-}$occupation building up after interband relaxation of the photoexcited electron-hole pair $[7,43]$. In the ideal case of complete interband relaxation, full inversion of the original absorption and a doubling of the normalized differential transmission to a value of $\Delta T / T=0.96 \times 10^{-3}$ would be expected. For the case at hand, the increase to the maximum signal level of about $\Delta T / T=0.80 \times 10^{-3}$ is characterized by a time 


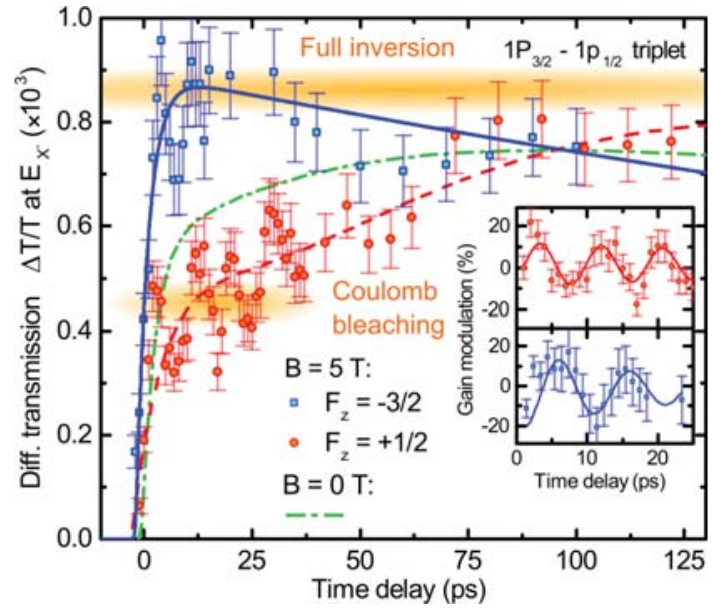

FIG. 6. Dynamics of the differential transmission signals on the $\mathrm{X}^{-}$resonance after selective excitation of the $F_{z}=+1 / 2$ (red circles, dashed line) and $-3 / 2$ (blue squares, solid line) triplet states. $\sigma^{+}$and $\sigma^{-}$polarized light is used for excitation, respectively. An external magnetic field of $B=5 \mathrm{~T}$ is applied in Faraday configuration to align the resident electron spin. The low-energy component of the Zeeman-split $\mathrm{X}^{-}$resonance is probed with $\sigma^{-}$-polarized photons. The two signal levels corresponding to bleaching and full gain are shaded with orange. Measurements are carried out at a temperature of $1.5 \mathrm{~K}$. The model fit of the data taken at $B=0 \mathrm{~T}$ (see Fig. 4, green dot-dashed line) is shown for comparison. The inset highlights oscillatory components extracted for time delays between 0 and $+30 \mathrm{ps}$, which are assigned to a spin procession of the excited trion caused by a slight discrepancy of magnetic field and optical readout directions in the photonic nanostructure.

constant of $(81 \pm 9) \mathrm{ps}$, as determined from the model fit. This value corresponds to a fractional gain which is to be statistically interpreted as a $(0.80 / 0.48-1)=0.67$ probability with which single-photon emission is stimulated $(81 \pm 9)$ ps after single-photon absorption at the initialized resonance. The fractional delayed gain results from a relatively inefficient intraband scattering of the $|+1 / 2\rangle_{1}$ state, involving a combined electron and hole spin flip induced by the coupling to the $|-3 / 2\rangle_{1}$ triplet via the EHX, as described in Sec. IV. In contrast, we expect a more efficient gain initialization when addressing the resonance which is directly associated with the $|-3 / 2\rangle_{1}$ state. Indeed, when exciting with a $\sigma^{-}$-polarized pump pulse, we observe a fast increase in the normalized differential transmission signal, reaching a plateau at a maximum value of $\Delta T / T=0.90 \times 10^{-3}$ (upper orange-shaded region). Remarkably, least-square fit analysis with our threelevel model indicates that we reach $\sigma^{-}$-polarized single-photon gain within $(2.8 \pm 0.5)$ ps and with near unity probability after an absorption of a pump photon. We also point out that the observed amplification amounts to approximately 1 stimulated photon per 1000 probe photons. This fact underlines the efficient coupling of our broadband femtosecond pulses to the QDs positioned inside the nanopillar structures.

By comparing the intraband relaxation of the $|+1 / 2\rangle_{1}$ and $|-3 / 2\rangle_{1}$ states at $B=5 \mathrm{~T}$ with that find for $B=0 \mathrm{~T}$ (green dashed line), we find a strikingly clear conservation rule, where the average of the individual measurements under the magnetic field coincides with the dynamical trace at $B=0 \mathrm{~T}$.
Such dependence is expected in the limit of applicability of the rate equation model, i.e., when polarization coherence can be ignored. To this end, we focus our attention on the early time dynamics under the applied magnetic field. By subtracting the model fits from the data, we uncover an oscillatory behavior of the signal (inset of Fig. 6). The peak-to-peak value of single-photon gain is observed to be modulated by more than 30 percent after the excitation of both the $| \pm 1 / 2\rangle_{1}$ (red line) and $| \pm 3 / 2\rangle_{1}$ (blue line) triplet states. By fitting an exponentially damped sinusoidal function to the data, we extract oscillation frequencies of $(99 \pm 6)$ and $(115 \pm 12) \mathrm{GHz}$, respectively, and a decay time of approximately $50 \mathrm{ps}$. We attribute this coherent signal to a Larmor precession, which, due to the measurement geometry, must occur around an axis perpendicular to the optical $z$ axis. Thus the pronounced coherent signal reflects a slight modification of the input field by the metallic subwavelength nanophotonic structures around the QDs. Judging from the $\pi$ phase difference of the two signals in Fig. 6, it is interesting to note that we can initialize a Larmor precession of gain in the coherent regime. In future experiments this could enable the coherent control and readout of single electron or hole spins, as demonstrated in, e.g., Refs. [13-17,75], but performed with femtosecond accuracy and directly in transient transmission. Furthermore, by employing a three-level lambda scheme [76], the investigated quantum emitters present a route towards coherent manipulation of gain on ultrafast time scales approaching just few optical cycles.

\section{SUMMARY AND OUTLOOK}

We have studied the ultrafast intraband dynamics in single negatively charged CdSe/ZnSe QDs. Photoluminescence and photoluminescence excitation measurements were employed together with theoretical calculations to provide an unambiguous identification of the low-energy resonances associated with the discrete electronic states of the system. Based on this input, we investigated the relaxation processes of specific excited states via time- and spectrally resolved two-color pump-probe measurements with femtosecond time resolution. Differential transmission signals in the vicinity of the fundamental trion resonance $\left(\mathrm{X}^{-}\right)$were monitored. In this way, we access detailed information on the intraband scattering processes within the manifold of identified few-particle configurations. Comparison of several QDs of different dimensions demonstrates that the gain onset time increases nonlinearly with the excess energy dissipated by the hole after pumping a transition from the valence-band $D$ shell into the $s$ shell of the conduction band. Consequently, we were able to establish single-photon gain at the $\mathrm{X}^{-}$resonance of a single quantum emitter on a time scale as fast as $780 \mathrm{fs}$. In contrast, excitation of $p$-shell transitions results in intraband relaxation of both electron and hole. In case of a singlet excitation, scattering into the trion ground state necessitates no spin flip. Consequently, a fast rise time of $\mathrm{X}^{-}$gain in the order of a few ps is found. Both the $D s$ and $P p$ singlet transitions exhibit moderate absorption, requiring relatively strong excitation in order to create an electron-hole pair. The most efficient way to establish maximum gain at the $\mathrm{X}^{-}$transition is excitation of the $P p$ triplet resonance. However, intraband relaxation following this pumping scheme might be slow due to the spin flip, which is required. Indeed, a 
ground-state exciton is generated only within approximately 50 ps when triplet states with $F_{z}= \pm 1 / 2$ are excited. Surprisingly, pumping of a resonance with $F_{z}= \pm 3 / 2$ results in inversion of the QD within a few picoseconds. In this case, the strong mixing of the triplet state with the excited-state singlet of the trion caused by the electron-electron exchange interaction removes the necessity of a spin flip for the relaxation to the XGS. While this configuration enables us to initialize single-photon gain on ultrafast time scales, the overall probability of the amplification process is still compromised by the stochastic spin distribution of the resident electron. Therefore it is advantageous to work in an external magnetic field of $5 \mathrm{~T}$. By implementing circular polarizations for the excitation and readout pulses, we were even able to deterministically select between relaxation of the excited trion triplet state with a time constant of 2.8 or $81 \mathrm{ps}$. In the former case, maximum gain provided by the QD is established in the most effective way. An overall efficiency of the amplifier of $0.1 \%$ is supported by the photonic nanostructure around the quantum emitters.

As an outlook, our characterization of few-particle dynamics in $\mathrm{CdSe} / \mathrm{ZnSe}$ QDs paves a way towards designing broadband quantum amplifiers for novel applications in ultrafast quantum optics and quantum information technology. By exploiting colloidal QDs [77-79], a new level of control over the position of the quantum emitters may be achieved via nanomanipulation procedures using, e.g., an AFM tip. This

[1] S. Rahimi-Keshari, T. C. Ralph, and C. M. Caves, Sufficient Conditions for Efficient Classical Simulation of Quantum Optics, Phys. Rev. X 6, 021039 (2016).

[2] G. S. Agarwal and K. Tara, Nonclassical properties of states generated by the excitations on a coherent state, Phys. Rev. A 43, 492 (1991).

[3] A. Zavatta, S. Viciani, and M. Bellini, Quantum-to-classical transition with single-photon-added coherent states of light, Science 306, 660 (2004).

[4] S. Rahimi-Keshari, T. Kiesel, W. Vogel, S. Grandi, A. Zavatta, and M. Bellini, Quantum Process Nonclassicality, Phys. Rev. Lett. 110, 160401 (2013).

[5] A. Zavatta, J. Fiurášek, and M. Bellini, A high-fidelity noiseless amplifier for quantum light states, Nat. Photonics 5, 52 (2011).

[6] R. Kumar, E. Barrios, C. Kupchak, and A. I. Lvovsky, Experimental Characterization of Bosonic Creation and Annihilation Operators, Phys. Rev. Lett. 110, 130403 (2013).

[7] F. Sotier, T. Thomay, T. Hanke, J. Korger, S. Mahapatra, A. Frey, K. Brunner, R. Bratschitsch, and A. Leitenstorfer, Femtosecond fewfermion dynamics and deterministic single-photon gain in a quantum dot, Nat. Phys. 5, 352 (2009).

[8] U. Woggon, Optical Properties of Semiconductor Quantum Dots (Springer-VerlagBerlin Heidelberg, 1997).

[9] V. Klimov, Nanocrystal Quantum Dots, 2nd ed. (CRC Press, 2010).

[10] A. Tartakovskii, Quantum Dots: Optics, Electron Transport and Future Applications (Cambridge University Press, 2012).

[11] I. A. Akimov, A. Hundt, T. Flissikowski, and F. Henneberger, Fine structure of the trion triplet state in a single self-assembled semiconductor quantum dot, Appl. Phys. Lett. 81, 4730 (2002). step could enable maximally efficient coupling of the quantum emitters to more sophisticated plasmonic nanostructures or nanoresonators [80]. Such hybrid systems involving single nanocrystals and advanced nanophotonic structures give rise to large enhancements in light-matter interaction, decreasing the amount of probe photons needed for extraction of ultrafast single-photon gain. Additionally, stronger light-matter coupling and broadband readout potentially enable operation at higher temperatures. Ultimately, differential transmission signals in the order of unity might be reached in this way, corresponding to probe pulses containing only one photon stimulating the emission of a second photon [81]. Such a system would, e.g., enable the deterministic creation of biphotons and eventually ultrashort wavepackets with well-defined photon numbers via multiple single-photon amplification processes. Also, working with QDs resonant to the mid-infrared spectral range would provide ultrafast emitters and amplifiers producing nonclassical states of light that could be directly read out in the time domain with ultrabroadband quantum sampling of the electric field [82-84].

\section{ACKNOWLEDGMENT}

Support by the Deutsche Forschungsgemeinschaft (DFG) via collaborative research center SFB767 is gratefully acknowledged.

[12] P. Michler, Quantum Dots for Quantum Information Technologies (Springer, 2017).

[13] J. Berezovsky, M. H. Mikkelsen, N. G. Stoltz, L. A. Coldren, and D. D. Awschalom, Picosecond coherent optical manipulation of a single electron spin in a quantum dot, Science 320, 349 (2008).

[14] D. Press, T. D. Ladd, B. Zhang, and Y. Yamamoto, Complete quantum control of a single quantum dot spin using ultrafast optical pulses, Nature (London) 456, 218 (2008).

[15] A. Greilich, S. E. Economou, S. Spatzek, D. R. Yakovlev, D. Reuter, A. D. Wieck, T. L. Reinecke, and M. Bayer, Ultrafast optical rotations of electron spins in quantum dots, Nat. Phys. 5, 262 (2009).

[16] K. Müller, A. Bechtold, C. Ruppert, C. Hautmann, J. S. Wildmann, T. Kaldewey, M. Bichler, H. J. Krenner, G. Abstreiter, M. Betz, and J. J. Finley, High-fidelity optical preparation and coherent Larmor precession of a single hole in an (In, Ga)As quantum dot molecule, Phys. Rev. B 85, 241306 (2012).

[17] Y. Kodriano, I. Schwartz, E. Poem, Y. Benny, R. Presman, T. A. Truong, P. M. Petroff, and D. Gershoni, Complete control of a matter qubit using a single picosecond laser pulse, Phys. Rev. B 85, 241304(R) (2012).

[18] T. Grange, E. A. Zibik, R. Ferreira, G. Bastard, B. A. Carpenter, P. J. Phillips, D. Stehr, S. Winnerl, M. Helm, M. J. Steer, M. Hopkinson, J. W. Cockburn, M. S. Skolnick, and L. R. Wilson, Singlet and triplet polaron relaxation in doubly charged selfassembled quantum dots, New J. Phys. 9, 259 (2007).

[19] E. A. Zibik, T. Grange, B. A. Carpenter, N. E. Porter, R. Ferreira, G. Bastard, D. Stehr, S. Winnerl, M. Helm, H. Y. Liu, M. S. Skolnick, and L. R. Wilson, Long lifetimes of quantum-dot 
intersublevel transitions in the terahertz range, Nat. Mater. 8, 803 (2009).

[20] W. Langbein, V. Cesari, F. Masia, A. B. Krysa, P. Borri, and P. M. Smowton, Ultrafast gain dynamics in InP quantum-dot optical amplifiers, Appl. Phys. Lett. 97, 211103 (2010).

[21] T. Guenther, C. Lienau, T. Elsaesser, M. Glanemann, V. M. Axt, T. Kuhn, S. Eshlaghi, and A. D. Wieck, Coherent Nonlinear Optical Response of Single Quantum Dots Studied by Ultrafast Near-Field Spectroscopy, Phys. Rev. Lett. 89, 057401 (2002).

[22] E. D. Kim, K. Truex, Y. Wu, A. Amo, X. Xu, D. G. Steel, A. S. Bracker, D. Gammon, and L. J. Sham, Picosecond optical spectroscopy of a single negatively charged self-assembled InAs quantum dot, Appl. Phys. Lett. 97, 113110 (2010).

[23] M. Zecherle, C. Ruppert, E. C. Clark, G. Abstreiter, J. J. Finley, and M. Betz, Ultrafast few-fermion optoelectronics in a single self-assembled InGaAs/GaAs quantum dot. Phys. Rev. B 82, 125314 (2010).

[24] C. Wolpert, C. Dicken, P. Atkinson, L. Wang, A. Rastelli, O. G. Schmidt, H. Giessen, and M. Lippitz, Transient reflection: A versatile technique for ultrafast spectroscopy of a single quantum dot in complex environments, Nano Lett. 12, 453 (2012).

[25] Q. Mermillod, D. Wigger, V. Delmonte, D. E. Reiter, C. Schneider, M. Kamp, S. Höfling, W. Langbein, T. Kuhn, G. Nogues, and J. Kasprzak, Dynamics of excitons in individual InAs quantum dots revealed in four-wave mixing spectroscopy, Optica 3, 377 (2016).

[26] T. Kaldewey, S. Lüker, A. V. Kuhlmann, S. R. Valentin, A. Ludwig, A. D. Wieck, D. E. Reiter, T. Kuhn, and R. J. Warburton, Coherent and robust high-fidelity generation of a biexciton in a quantum dot by rapid adiabatic passage, Phys. Rev. B 95, 161302(R) (2017).

[27] L. Robledo, J. Elzerman, G. Jundt, M. Atatüre, A. Högele, S. Fält, and A. Imamoglu, Conditional dynamics of interacting quantum dots, Science 320, 772 (2008).

[28] A. Srivastava, M. Sidler, A. V. Allain, D. S. Lembke, A. Kis, and A. Imamoğlu, Optically active quantum dots in monolayer $\mathrm{WSe}_{2}$, Nat. Nanotechnol. 10, 491 (2015).

[29] M. Koperski, K. Nogajewski, A. Arora1, V. Cherkez, P. Mallet, J.-Y. Veuillen, J. Marcus, P. Kossacki, and M. Potemski, Single photon emitters in exfoliated $\mathrm{WSe}_{2}$ structures, Nat. Nanotechnol. 10, 503 (2015).

[30] P. Tonndorf, R. Schmidt, R. Schneider, J. Kern, M. Buscema, G. A. Steele, A. Castellanos-Gomez, H. S. J. Van Der Zant, S. Michaelis de Vasconcellos, and R. Bratschitsch, Singlephoton emission from localized excitons in an atomically thin semiconductor, Optica 2, 347 (2015).

[31] A. Kormányos, V. Zólyomi, N. D. Drummond, and G. Burkard, Spin-Orbit Coupling, Quantum Dots, and Qubits in Monolayer Transition Metal Dichalcogenides, Phys. Rev. X 4, 011034 (2014).

[32] O. Zakharov, A. Rubio, X. Blase, M. L. Cohen, and S. G. Louie, Quasi-particle band structures of 6 II-VI-compounds: ZnS, ZnSe, ZnTe, CdS, CdSe, and CdTe, Phys. Rev. B 50, 10780 (1994).

[33] O. Madelung, Semiconductors: Data Handbook (Springer, 2004).

[34] T. Kazimierczuk, T. Smoleński, J. Kobak, M. Goryca, W. Pacuski, A. Golnik, K. Fronc, P. Wojnar, and P. Kossacki, Optical study of electron-electron exchange interaction in CdTe/ZnTe quantum dots, Phys. Rev. B 87, 195302 (2013).
[35] T. Smoleński, T. Kazimierczuk, M. Goryca, P. Wojnar, and P. Kossacki, Fine structure of a resonantly excited p-shell exciton in a CdTe quantum dot, Phys. Rev. B 93, 195311 (2016).

[36] U. Woggon, H. Giessen, F. Gindele, and O. Wind, B. Fluegel and N. Peyghambarian, Ultrafast energy relaxation in quantum dots, Phys. Rev. B 54, 17681 (1996).

[37] V. I. Klimov and D. W. McBranch, Femtosecond 1P-to-1S Electron Relaxation in Strongly Confined Semiconductor Nanocrystals, Phys. Rev. Lett. 80, 4028 (1998).

[38] V. I. Klimov, D. W. McBranch, C. A. Leatherdale, and M. G. Bawendi, Electron and hole relaxation Pathways in semiconductor quantum dots, Phys. Rev. B 60, 13740 (1999).

[39] P. Guyot-Sionnest, M. Shim, C. Matranga, and M. Hines, Intraband relaxation in CdSe quantum dots, Phys. Rev. B 60, 2181(R) (1999).

[40] C. Burda, S. Link, M. Mohamed, and M. El-Sayed, The relaxation pathways of $\mathrm{CdSe}$ nanoparticles monitored with femtosecond time-resolution from the visible to the IR: Assignment of the transient features by carrier quanching, J. Phys. Chem. B 105, 12286 (2001).

[41] P. Peng, D. J. Milliron, S. M. Hughes, J. C. Johnson, A. P. Alivisatos, and R. J. Saykally, Femtosecond spectroscopy of carrier relaxation dynamics in type II CdSe/CdTe tetrapod heteronanostructures, Nano Lett. 5, 1809 (2005).

[42] J. M. An, M. Califano, A. Franceschetti, and A. Zunger, Excitedstate relaxation in PbSe quantum dots, J. Chem. Phys. 128, 164720 (2008).

[43] J. Huneke, I. D’Amico, P. Machnikowski, T. Thomay, R. Bratschitsch, A. Leitenstorfer, and T. Kuhn, Role of Coulomb correlations for femtosecond pump-probe signals obtained from a single quantum dot, Phys. Rev. B 84, 115320 (2011).

[44] L. De Santis, C. Antón, B. Reznychenko, N. Somaschi, G. Coppola, J. Senellart, C. Gómez, A. Lemaître, I. Sagnes, A. G. White, L. Lanco, A. Auffèves, and P. Senellart, A solid-state single photon filter, Nat. Nanotechnol. 12, 663 (2017).

[45] S. Mahapatra, K. Brunner, and C. Bougerol, Self-assembly of $\mathrm{CdSe} / \mathrm{ZnSe}(001)$ quantum dot structures mediated by a tellurium cap layer, Appl. Phys. Lett. 91, 153110 (2007).

[46] C. Genet and T. W. Ebbesen, Light in tiny holes, Nature (London) 445, 39 (2007).

[47] H. F. Ghaemi, T. Thio, D. E. Grupp, T. W. Ebbesen, and H. J. Lezec, Surface plasmons enhance optical transmission through subwavelength holes, Phys. Rev. B 58, 6779 (1998).

[48] D. Brida, G. Krauss, A. Sell, and A. Leitenstorfer, Ultrabroadband Er: Fiber lasers, Laser Photon. Rev. 8, 409 (2014).

[49] Al. L. Efros and M. Rosen, Band-edge exciton in quantum dots of semiconductors with a degenerate valence band: Dark and bright exciton state, Phys. Rev. B 54, 4843 (1996).

[50] M. Bayer, G. Ortner, O. Stern, A. Kuther, A. A. Gorbunov, A. Forchel, P. Hawrylak, S. Fafard, K. Hinzer, T. L. Reinecke, S. N. Walck, J. P. Reithmaier, F. Klopf, and F. Schäfer, Fine structure of neutral and charged excitons in self-assembled In(Ga)As/(Al)GaAs quantum dots, Phys. Rev. B 65, 195315 (2002).

[51] K. V. Kavokin, Fine structure of the quantum-dot trion, Phys. Stat. Sol.(a) 195, 592 (2003).

[52] I. A. Akimov, T. Flissikowski, A. Hundt, and F. Henneberger, Spin processes related to trions in quantum dots, Phys. Stat. Sol.(a) 201, 412 (2004). 
[53] V. D. Glazov, and M. M. Kulakovskii, Spin-orbit effect on electron-electron interaction and the fine structure of electron complexes in quantum dots, Phys. Rev. B 79, 195305 (2009).

[54] I. A. Akimov, K. V. Kavokin, A. Hundt, and F. Henneberger, Electron-hole exchange interaction in a negatively charged quantum dot, Phys. Rev. B 71, 075326 (2005).

[55] M. E. Ware, E. A. Stinaff, D. Gammon, M. F. Doty, A. S. Bracker, D. Gershoni, V. L. Korenev, S. C. Badescu, Y. Lyanda-Geller, and T. L. Reinecke, Polarized Fine Structure in the Photoluminescence Excitation Spectrum of a Negatively Charged Quantum Dot, Phys. Rev. Lett. 95, 177403 (2005).

[56] V. D. Kulakovskii, G. Bacher, R. Weigand, T. Kummell, A. Forchel, E. Borovitskaya, K. Leonardi, and D. Hommel, Fine Structure of Biexciton Emission in Symmetric and Asymmetric CdSe/ZnSe Single Quantum Dots, Phys. Rev. Lett. 82, 1780 (1999).

[57] A. I. Ekimov, I. A. Kudryavtsev, A. L. Efros, T. V. Yazeva, F. Hache, M. C. Schanne-Klein, A. V. Rodina, D. Ricard, and C. Flytzanis, Absorption and intensity-dependent photoluminescence measurements on CdSe quantum dots: Assignment of the first electronic transitions, J. Opt. Soc. Am. B 10, 100 (1993).

[58] U. E. H. Laheld and G. T. Einevoll, Excitons in CdSe quantum dots, Phys. Rev. B 55, 5184 (1997).

[59] G. V. Astakhov, T. Kiessling, A. V. Platonov, T. Slobodskyy, S. Mahapatra, W. Ossau, G. Schmidt, K. Brunner, and L. W. Molenkamp, Circular-to-Linear and Linear-to-Circular Conversion of Optical Polarization by Semiconductor Quantum Dots, Phys. Rev. Lett. 96, 027402 (2006).

[60] T. Takagahara, Theory of exciton doublet structures and polarization relaxation in single quantum dots, Phys. Rev. B 62, 16840 (2000).

[61] E. Kadantsev and P. Hawrylak, Theory of exciton fine structure in semiconductor quantum dots: Quantum dot anisotropy and lateral electric field, Phys. Rev. B 81, 045311 (2010).

[62] J. F. Harvey, H. Shen, R. A. Lux, M. Dutita, J. Pamulapati, and R. Tsu, Raman and optical characterization of porous silicon, Mater. Res. Soc. Symp. Proc. 256, 175 (1991).

[63] L.-W. Wang and Alex Zunger, Dielectric Constants of Silicon Quantum Dots, Phys. Rev. Lett. 73, 1039 (1994).

[64] R. Tsu, D. Babic, and L. Ioriatti, Jr., Simple model for the dielectric constant of nanoscale silicon particle, J. Appl. Phys. 82, 1327 (1997).

[65] M. Nirmal, D. J. Norris, M. Kuno, M. G. Bawendi, A. L. Efros, and M. Rosen, Observation of the "Dark Exciton" in CdSe Quantum Dots, Phys. Rev. Lett. 75, 3728 (1995).

[66] A. Wojs and P. Hawrylak, Negatively charged magnetoexcitons in quantum dots, Phys. Rev. B 51, 10880 (1995).

[67] O. Verzelen, R. Ferreira, and G. Bastard, Excitonic Polarons in Semiconductor Quantum Dots, Phys. Rev. Lett. 88, 146803 (2002).

[68] S. S. Mitra, Phonon assignments in $\mathrm{ZnSe}$ and $\mathrm{GaSb}$ and some regularities in the phonon frequencies of zincblende-type semiconductors, Phys. Rev. 132, 986 (1963).

[69] S. C. Badescu, Y. Lyanda-Geller, and T. L. Reinecke, Asymmetric exchange between electron spins in coupled semiconductor quantum dots, Phys. Rev. B 72, 161304 (2005).
[70] S. Cortez, O. Krebs, S. Laurent, M. Senes, X. Marie, P. Voisin, R. Ferreira, G. Bastard, J.-M. Gerard, and T. Amand, Optically Driven Spin Memory in $n$-Doped InAs-GaAs Quantum Dots, Phys. Rev. Lett. 89, 207401 (2002).

[71] Y. Benny, R. Presman, Y. Kodriano, E. Poem, and D. Gershoni, Electron-hole spin flip-flop in semiconductor quantum dots, Phys. Rev. B 89, 035316 (2014).

[72] A. V. Khaetskii and Y. V. Nazarov, Spin relaxation in semiconductor quantum dots, Phys. Rev. B 61, 12639 (2000).

[73] F. Liu, L. Biadala, A. V. Rodina, D. R. Yakovlev, D. Dunker, C. Javaux, J.-P. Hermier, A. L. Efros, B. Dubertret, and M. Bayer, Spin dynamics of negatively charged excitons in $\mathrm{CdSe} / \mathrm{CdS}$ colloidal nanocrystals, Phys. Rev. B 88, 035302 (2013).

[74] E. G. Kavousanaki and G. Burkard, Signatures of spin blockade in the optical response of a charged quantum dot, Phys. Rev. B 86, 045122 (2012).

[75] T. M. Godden, J. H. Quilter, A. J. Ramsay, Y. Wu, P. Brereton, S. J. Boyle, I. J. Luxmoore, J. Puebla-Nunez, A. M. Fox, and M. S. Skolnick, Coherent Optical Control of the Spin of a Single Hole in an InAs/GaAs Quantum Dot, Phys. Rev. Lett. 108, 017402 (2012).

[76] X. Xu, B. Sun, P. R. Berman, D. G. Steel, A. S. Bracker, D. Gammon, and L. J. Sham, Coherent population trapping of an electron spin in a single negatively charged quantum dot, Nat. Phys. 4, 692 (2008).

[77] C. Negele, J. Haase, A. Budweg, A. Leitenstorfer, and S. Mecking, Stable single-photon emission by quantum dot/polymer hybrid particles, Macromol. Rapid Commun. 34, 1145 (2013).

[78] T. de Roo, J. Haase, J. Keller, C. Hinz, M. Schmid, D.V. Seletskiy, H. Cölfen, A. Leitenstorfer, and S. Mecking, A direct approach to organic/inorganic semiconductor hybrid particles via functionalized polyfluorene ligands, Adv. Funct. Mater. 24, 2714 (2014).

[79] F. Werschler, C. Hinz, F. Froning, P. Gumbsheimer, J. Haase, C. Negele, T. de Roo, S. Mecking, A. Leitenstorfer, and D. V. Seletskiy, Coupling of excitons and discrete acoustic phonons in vibrationally isolated quantum emitters, Nano Lett. 16, 5861 (2016).

[80] W.-M. Schulz, T. Thomay, M. Eichfelder, M. Bommer, M. Wiesner, R. Roßbach, M. Jetter, R. Bratschitsch, A. Leitenstorfer, and P. Michler, Optical properties of red emitting self-assembled InP/(Al0.20Ga0.80)0.51In0.49P quantum dot based micropillars, Opt. Express 18, 12543 (2010).

[81] G. Wrigge, I. Gerhardt, J. Hwang, G. Zumofen, and V. Sandoghdar, Efficient coupling of photons to a single molecule and the observation of its resonance fluorescence, Nat. Phys. 4, 60 (2008).

[82] C. Riek, D. V. Seletskiy, A. S. Moskalenko, J. F. Schmidt, P. Krauspe, S. Eckart, S. Eggert, G. Burkard, and A. Leitenstorfer, Direct sampling of electric-field vacuum fluctuations, Science 350, 420 (2015).

[83] A. S. Moskalenko, C. Riek, D. V. Seletskiy, G. Burkard, and A. Leitenstorfer, Paraxial Theory of Direct Electro-optic Sampling of the Quantum Vacuum, Phys. Rev. Lett. 115, 263601 (2015).

[84] C. Riek, P. Sulzer, M. Seeger, A. S. Moskalenko, G. Burkard, D. V. Seletskiy, and A. Leitenstorfer, Subcycle quantum electrodynamics, Nature (London) 541, 376 (2017). 